$12-1-2019$

\title{
On Vibration Suppression and Trajectory Tracking in Largely Uncertain Torsional System: An Error-based ADRC Approach
}

\author{
R. Madonski \\ Southeast University \\ M. Ramirez-Neria \\ Universidad Politécnica del Valle de México \\ M. Stankovic \\ University of Defense \\ Sally Shao \\ Cleveland State University, s.shao@csuohio.edu \\ Zhiqiang Gao \\ Cleveland State University, Z.GAO@csuohio.edu
}

See next page for additional authors

Follow this and additional works at: https://engagedscholarship.csuohio.edu/scimath_facpub

Part of the Mathematics Commons

How does access to this work benefit you? Let us know!

\section{Repository Citation}

Madonski, R.; Ramirez-Neria, M.; Stankovic, M.; Shao, Sally; Gao, Zhiqiang; Yang, J.; and Li, S., "On Vibration Suppression and Trajectory Tracking in Largely Uncertain Torsional System: An Error-based ADRC Approach" (2019). Mathematics Faculty Publications. 317.

https://engagedscholarship.csuohio.edu/scimath_facpub/317

This Article is brought to you for free and open access by the Mathematics and Statistics Department at EngagedScholarship@CSU. It has been accepted for inclusion in Mathematics Faculty Publications by an authorized administrator of EngagedScholarship@CSU. For more information, please contact library.es@csuohio.edu. 


\section{Authors}

R. Madonski, M. Ramirez-Neria, M. Stankovic, Sally Shao, Zhiqiang Gao, J. Yang, and S. Li 


\section{On vibration suppression and trajectory tracking in largely uncertain torsional system: An error-based ADRC approach}

R. Madonski, M. Ramirez-Neria, M. Stanković, S. Shao, Z. Gao, J. Yang, S. Li

A R T I C L E I N F O

Article history:

Received 10 June 2019

Received in revised form 14 July 2019

Accepted 8 August 2019

Available online 29 August 2019

Keywords:

Active disturbance rejection control

Extended state observer

Vibration suppression

Harmonic reference tracking

Harmonic disturbance rejection

Torsional plant

\section{Introduction}

The compensation of harmonic uncertainty is an important, yet challenging, task in control engineering. Its practical significance is noticeable, for example, in suppressing vibration in mass-damper systems [1,2], controlling overhead cranes $[3,4]$ as well as in energy-harvesting systems $[5,6]$. Throughout the years, numerous approaches have been developed in academia to address the problem with some representative examples [7-11].

This work, however, focuses solely on the developments coming from the area of active disturbance rejection control (ADRC [12]) methodology. The justification of such deliberate exclusion comes from the fact that the ADRC-based techniques have recently became a serious alternative to conventional industrial controllers (like the omnipresent PID). With a trackrecord of successful deployments in power [13,14], motion [15-18], and process [19,20] control settings, the ADRC-based methods have been verified to bring significant enhancement to the usual PID control performance (in terms of accuracy, 
repeatability, and/or energy efficiency), while retaining its implementation simplicity and intuitiveness of each control term. What is more, the ADRC-based techniques are the few approaches that made a successful transition from academia to industry and are now incorporated by control software and instrumentation manufacturers (like Texas Instruments or Danfoss) in form of off-the-shelf motor and process control solutions. Hence, their commercial success motivates to find new ADRCinspired solutions to well-known problems in order to find, not only quantitative improvements, but also qualitative ones. For these reasons, the treated problem of harmonic uncertainty is reformulated here to be a disturbance-rejection problem, from which a novel, creative, and practically appealing solution is conceived.

The problem of harmonic uncertainty compensation have been studied before in the context of ADRC with representable works [21-27]. From analyzing them, the usual approach is to design an output-based two-stage ADRC structure, where the first stage is responsible for real-time estimation and rejection of the generalized disturbance, and the other stage governs the resultant (theoretically disturbance-free) system dynamics in accordance to the given control objective. And although such two degree-of-freedom (2DOF) composition has its advantages in academic deliberations (like the ability to design, tune, and analyze the two stages separately), it may not always be desirable in practice, which favors simplicity of having a single, compact structure, explicitly expressing the feedback error-to-control signal channel. Furthermore, the majority of industrial controllers (lead by PID) are designed in explicit 1DOF feedback error-based form, which is easily understood and handled by the industry staff. Hence, in order to practically tackle the problem of harmonic uncertainty, the need is to reformulate the typical 2DOF output-based ADRC and express it in a more industry-familiar PID-like error-based form, straightforwardly deployable across different industrial platforms, without losing its on-line disturbance rejection capabilities.

Hence, in it this work, an error-based 1DOF ADRC is introduced and customized to handle multiple sources of disturbances, including harmonic ones. A modified, error-based version of the recently introduced resonant extended state observer (RESO [27]) is used as the core component in the new ADRC to deal with various sources of uncertainties, including those being the sinusoidal character of the target signal and/or the sinusoidal influence of external factors on the governed plant. The proposed approach simplifies the original output-based formulation and reduces its application restrictions making the improved control method as convenient as PID in its commission. The specific contributions of this work are thus as follows:

(i) introduction of a new, customized, error-based ADRC control scheme capable of effectively compensating complex (including harmonic) uncertainties;

(ii) its rigorous stability proof using singular perturbation theory;

(iii) its tracking performance and disturbance rejection analysis in frequency domain;

(iv) and its multi-criteria experimental validation on a challenging laboratory testbed.

The proposed control topology, although applicable to a larger class of systems, will be introduced and exemplified throughout this work using solely the case of a 3DOF torsional mechanism, which is well-known and representable model of vibration in many mechanical systems.

\section{Preliminaries}

\subsection{Plant physical and design models}

The considered torsional system, seen in Fig. 1, consists of three discs suspended vertically on an anti-friction ball bearings mounted on a metal structure. The discs are connected through two torsional springs with positive coefficients $\kappa_{1}, \kappa_{2}$. The second (middle) and third (top) discs are considered to be the load of the first (bottom) disc, which is driven (actuated) with an electric motor. Signals $\theta_{1}, \theta_{2}$, and $\theta_{3}$ denote the angular positions of first, second, and third disc, respectively. The total inertia of each corresponding disc is: $J_{1}=J_{d}+J_{m}+2 m r^{2}, J_{2}=J_{d}+2 m r^{2}$, and $J_{3}=J_{d}+2 m r^{2}$. Extra two masses are attached to each plate, where $m$ is the weight of each mass, $r$ is the distance of the mass to the center of the disc, $J_{m}$ is the motor inertia, and $J_{d}$ is the plate inertia. Term $\tau$ represents the control input (torque) to the system. A simplified design model of the considered torsional mechanism can be thus obtained as [28]:

$$
\begin{aligned}
& J_{1} \ddot{\theta}_{1}+\kappa_{1} \theta_{1}-\kappa_{1} \theta_{2}=\tau, \\
& J_{2} \ddot{\theta}_{2}-\kappa_{1} \theta_{1}+\left(\kappa_{1}+\kappa_{2}\right) \theta_{2}-\kappa_{2} \theta_{3}=0, \\
& J_{3} \ddot{\theta}_{3}-\kappa_{2} \theta_{2}+\kappa_{2} \theta_{3}=0 .
\end{aligned}
$$

Unlike the idealized design plant model (1), a real torsional mechanism (with its subsequent physical model) is a challenging control problem subjected to multiple sources of uncertainties, like friction in the motor and pulleys, amplifier nonlinearities, vibration modes, sensor noise, actuator saturation, and torque ripple in the brushless motor commutation (up to 13\%). Some of them are reviewed briefly below to show the complexity of the real mechanism and the amount of uncertainty that the control system will have to deal with. ${ }^{1}$

\footnotetext{
${ }^{1}$ A distinction is made here between a physical model and a design model of the torsional plant. The former comes from physics and encapsulates the acting physical phenomena. The latter is a simple, useful representation of the governed plant for the purpose of a straightforward control formulation.
} 

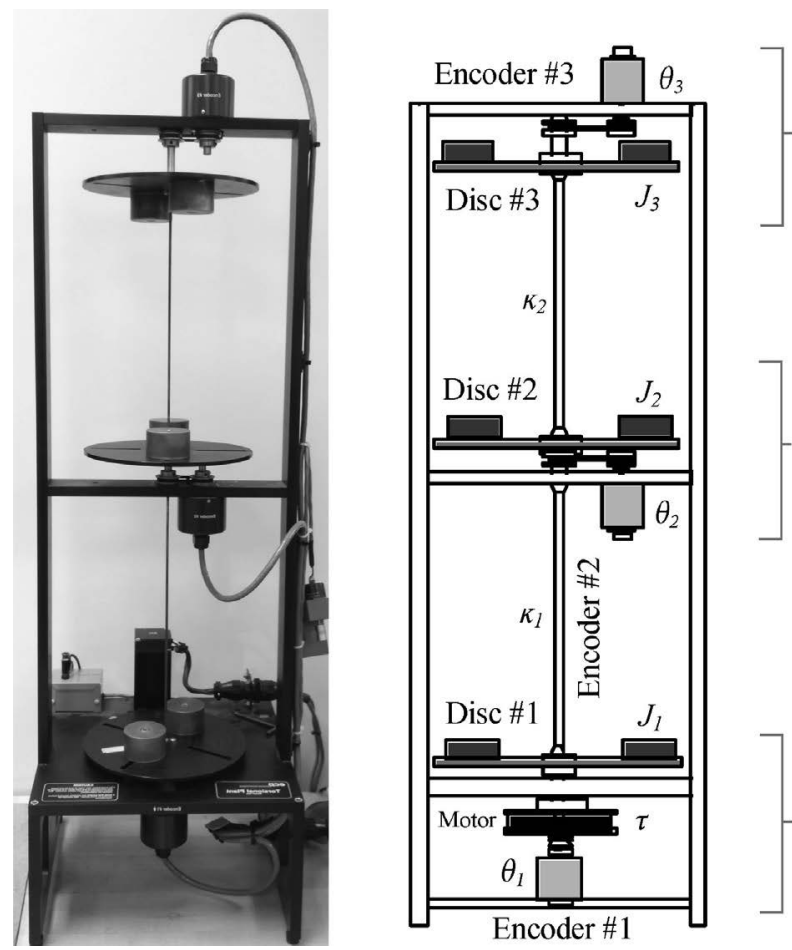

Underactuated, compliant dynamics

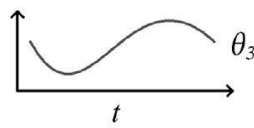

Underactuated, compliant dynamics

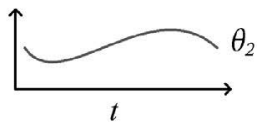

Actuated, rigid dynamics

Fig. 1. Real system (left) and its simplified diagram (right)

\subsubsection{Unmodeled dynamics}

While (1) is useful in capturing the predominant dynamic plant behavior, it purposefully neglects certain non-ideal effects. Among these are electrical part (associated with input torque generation), torques associated with motor cogging, Coulomb (or static) and viscous friction from the pulleys and bearings. Upon Fig. 2, a more precise approximation of the torsional system is:

$$
\begin{aligned}
& J_{1} \ddot{\theta}_{1}+\overbrace{\bar{c}_{v 1} \dot{\theta}_{1}+\bar{k}_{c} \sin \left(n \theta_{1}+\phi\right)+\bar{c}_{s 1} \operatorname{sign}\left(\dot{\theta}_{1}\right)}^{f_{\text {ud } 1}}+\kappa_{1} \theta_{1}-\kappa_{1} \theta_{2}=\tau, \\
& J_{2} \ddot{\theta}_{2}+\overbrace{\bar{c}_{v 2} \dot{\theta}_{2}+\bar{c}_{s 2} \operatorname{sign}\left(\dot{\theta}_{2}\right)}^{f_{\text {ud } 2}}-\kappa_{1} \theta_{1}+\left(\kappa_{1}+\kappa_{2}\right) \theta_{2}-\kappa_{2} \theta_{3}=0, \\
& J_{3} \ddot{\theta}_{3}+\overbrace{\bar{c}_{v 3} \dot{\theta}_{3}+\bar{c}_{\text {s3 }} \operatorname{sign}\left(\dot{\theta}_{3}\right)}^{f_{\text {ud } 3}}-\kappa_{2} \theta_{2}+\kappa_{2} \theta_{3}=0,
\end{aligned}
$$

where $\bar{c}_{s 1}-\bar{c}_{s 3}$ and $\bar{c}_{v 1}-\bar{c}_{v 3}$ are the Coulomb and viscous friction coefficients, respectively. While motor cogging is not a true sinusoid, it may be approximated by $\bar{k}_{c} \sin \left(n \theta_{1}+\phi\right)$, where $n$ is equal to the drive pulley diameter ratio times the number of motor poles (in the considered case $n=2 \times 3$ ). Terms $f_{\mathrm{ud} 1}, f_{\mathrm{ud} 2}$, and $f_{\mathrm{ud} 3}$ are the lumped unmodeled dynamic components of the first, second, and third equation in the above system, respectively.

\subsubsection{Parametric uncertainties}

Note, that even the simplified analytical representation of the plant requires measurement or estimation of several plant parameters, which may be physically difficult to obtain in practice. What is more, some of them may be also time-varying. For example, inertia (including both motor and load) increases with time in electric winding machine. It is thus reasonable to assume that a parametric modeling mismatch will exists when considering controlling a real system. For that reason the system can be modeled as:

$$
\begin{aligned}
& J_{01} \ddot{\theta}_{1}+\kappa_{01} \theta_{1}-\kappa_{01} \theta_{2}=\tau-f_{\mathrm{ud} 1}-f_{\mathrm{pu} 1}, \\
& J_{02} \ddot{\theta}_{2}-\kappa_{01} \theta_{1}+\left(\kappa_{01}+\kappa_{02}\right) \theta_{2}-\kappa_{02} \theta_{3}=-f_{\mathrm{ud} 2}-f_{\mathrm{pu} 2}, \\
& J_{03} \ddot{\theta}_{3}-\kappa_{02} \theta_{2}+\kappa_{02} \theta_{3}=-f_{\mathrm{ud} 3}-f_{\mathrm{pu} 3},
\end{aligned}
$$




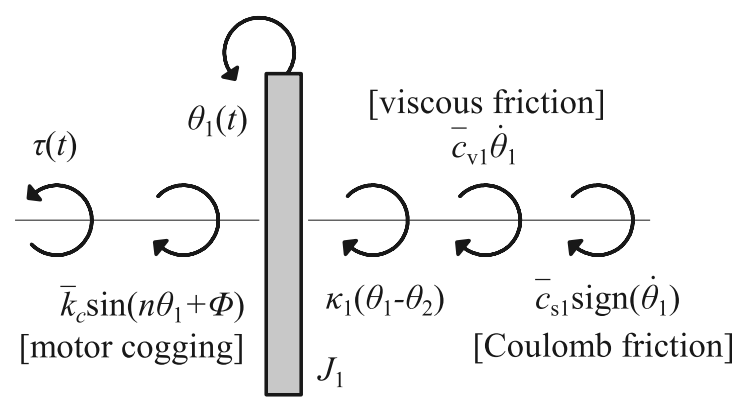

Fig. 2. Free body diagram considering friction and nonlinearities.

where $J_{01}, J_{02}, J_{03}, \kappa_{01}, \kappa_{02}$ are approximated values of corresponding parameters, while $f_{\mathrm{pu} 1}\left(J_{1}, \kappa_{1}, \kappa_{2}, \cdot\right), f_{\mathrm{pu} 2}\left(J_{2}, \kappa_{1}, \kappa_{2}, \cdot\right)$, and $f_{\mathrm{pu} 3}\left(J_{3}, \kappa_{2}, \cdot\right)$ represent the lumped parametric uncertainties of the first, second, and third equation in the above system, respectively.

\subsubsection{External disturbances}

The load torque is generally regarded as the most serious disturbance limiting performance. The angular position of the controlled disc will inevitably fluctuate when the load torque is imposed on (or displaced from) the motor. For that reason, an input-additive uncertainty in load torque can be added in the control channel as:

$$
J_{01} \ddot{\theta}_{1}+\kappa_{01} \theta_{1}-\kappa_{01} \theta_{2}=\tau-\overbrace{\tau_{\mathrm{ex}}-\tau_{\mathrm{lt}}}^{f_{\text {ed }}}-f_{\text {ud } 1}-f_{\text {pu1 }},
$$

where $f_{\text {ed }}$ is the overall torque disturbance, which consists of uncertainty in load torque $\left(\tau_{\mathrm{lt}}\right)$ and external input-additive torque disturbance $\left(\tau_{\mathrm{ex}}\right)$. Furthermore, in practice, the output measurement is inevitably corrupted with noise due to quantization of sensors. ${ }^{2}$

\subsubsection{Vibration modes}

The vibratory tendencies of the torsional plant, crucial in the context of this particular work, are best illustrated by considering a modal representation of the plant [29]. First, the system model (1) in matrix notation yields: $\boldsymbol{J} \boldsymbol{\theta}+\boldsymbol{k} \boldsymbol{\theta}=\boldsymbol{T}$, with $\boldsymbol{\theta}=\left[\theta_{1} \theta_{2} \theta_{3}\right]^{\top}$,

$$
\boldsymbol{k}=\left[\begin{array}{ccc}
\kappa_{1} & -\kappa_{1} & 0 \\
-\kappa_{1} & \kappa_{1}+\kappa_{2} & -\kappa_{2} \\
0 & -\kappa_{2} & \kappa_{2}
\end{array}\right], \quad J=\left[\begin{array}{ccc}
J_{1} & 0 & 0 \\
0 & J_{2} & 0 \\
0 & 0 & J_{3}
\end{array}\right],
$$

and $\boldsymbol{T}=[\tau 00]^{\top}$. The homogeneous $(\tau(t)=0)$ solutions of the above are harmonic and have a general form:

$$
\theta_{i}=\chi_{i} C \cos (\omega t+\phi), \quad i=1,2,3 .
$$

Substituting the above into (1) yields an eigenvalue problem:

$$
\left[\begin{array}{ccc}
\kappa_{1}-J_{1} \omega^{2} & -\kappa_{1} & 0 \\
-\kappa_{1} & \kappa_{1}+\kappa_{2}-J_{2} \omega^{2} & -\kappa_{2} \\
0 & -\kappa_{2} & \kappa_{2}-J_{3} \omega^{2}
\end{array}\right]\left\{\begin{array}{l}
\chi_{1} \\
\chi_{2} \\
\chi_{3}
\end{array}\right\}=0
$$

with characteristic equation:

$$
J_{1} J_{2} J_{3} \omega^{6}-\left[J_{1}\left(J_{2} \kappa_{2}+J_{3} \kappa_{1}+J_{3} \kappa_{2}\right)+J_{2} J_{3} \kappa_{1}\right] \omega^{4}+\left[J_{1} \kappa_{1} \kappa_{2}+J_{2} \kappa_{1} \kappa_{2}+J_{3} \kappa_{1} \kappa_{2}\right] \omega^{2}=0,
$$

which contains $\omega^{2}$ as a factor and hence a rigid body mode $(\omega=0)$ is present.

Remark 1. Given the above sources of different disturbances/uncertainties, the simplified representation (1) will be deliberately used for the upcoming observer and controller synthesis nevertheless and it is due to the underlying design principle behind ADRC, which states that as long as the overall, lumped uncertainty is compensated for in real time, an exact mathematical model of the plant is not required [30].

\subsection{Control objective}

A trajectory tracking task is assumed here, for which the system output $\left(\theta_{1}\right)$, being the angular position of the first disc manipulated by the control signal $(\tau)$, has to follow a smooth user-defined polynomial-type reference signal $\left(\theta_{1}^{*}\right)$. The goal is

\footnotetext{
${ }^{2}$ Direct use of noisy feedback signals can result in deterioration of the control performance which, in extreme cases, can lead to closed-loop instability.
} 
to track the target profile in absence of a detailed plant mathematical model for controller synthesis and in spite the influence of perturbations (including those of harmonic nature) acting on the plant output during its operation. The reference signal $\left(\theta_{1}^{*}\right)$ additionally satisfies following assumptions:

A1. it is bounded and known only at current time instant $t$,

A2. its consecutive time-derivatives $\left(\dot{\theta}_{1}^{*}, \ddot{\theta}_{1}^{*}\right)$ exist for all $t \geqslant 0$ and are bounded,

A3. it is not known in advance, nor its consecutive reference time-derivatives $\left(\dot{\theta}_{1}^{*}, \ddot{\theta}_{1}^{*}\right)$. In order to make the control solution more practical, additional assumptions are made:

A4. Output $\theta_{1}$ is the only one available for control synthesis.

A5. Structure (1) is known but its parameters are uncertain.

\section{Proposed error-based ADRC structure}

The proposed control solution consists of three distinctive design steps, namely: system transformation, controller design, and observer design. Following subsections document details of each step.

\subsection{System transformation}

Given the above control objective, it is beneficial to analyze first how the underactuated dynamics of the middle and top discs affects the actuated disc in the bottom and the governed output in result. In (1), substitution of second and third equations in the first one, leads to a following second order dynamics $(n=2)$ from the controlled output perspective:

$$
\ddot{\theta}_{1}=\frac{\tau(t)}{J_{1}}-\frac{\kappa_{1} \kappa_{2}}{J_{1}\left(\kappa_{1}+\kappa_{2}\right)} \theta_{1}+\frac{\kappa_{1} \kappa_{2}}{J_{1}\left(\kappa_{1}+\kappa_{2}\right)} \theta_{2}-\frac{J_{2} \kappa_{1}}{J_{1}\left(\kappa_{1}+\kappa_{2}\right)} \ddot{\theta}_{2}-\frac{J_{3} \kappa_{1}}{J_{1}\left(\kappa_{1}+\kappa_{2}\right)} \ddot{\theta}_{3}, J_{1} \neq 0 .
$$

The system (4) can be expressed alternatively as a causal input-output relation of a perturbed chain of integrators, for which ADRC schemes are most conveniently derived for, which yields:

$$
\ddot{\theta}_{1}=\tau(t) / J_{1}+\tilde{d}(t)
$$

where $\tilde{d}(t)$ is the internal disturbance, which lumps the effects of higher-order dynamics from middle and top discs:

$$
\tilde{d}(t)=-\frac{\kappa_{1} \kappa_{2}}{J_{1}\left(\kappa_{1}+\kappa_{2}\right)} \theta_{1}+\frac{\kappa_{1} \kappa_{2}}{J_{1}\left(\kappa_{1}+\kappa_{2}\right)} \theta_{2}-\frac{J_{2} \kappa_{1}}{J_{1}\left(\kappa_{1}+\kappa_{2}\right)} \ddot{\theta}_{2}-\frac{J_{3} \kappa_{1}}{J_{1}\left(\kappa_{1}+\kappa_{2}\right)} \ddot{\theta}_{3} .
$$

In order to solve the given trajectory tracking problem, tracking error is defined as: $e_{\theta_{1}} \triangleq \theta_{1}^{*}-\theta_{1}$, and, following the methodology independently developed in [31,32], the corresponding tracking error dynamics writes:

$$
\begin{aligned}
\ddot{e}_{\theta_{1}} & =\ddot{\theta}_{1}^{*}-\ddot{\theta}_{1}, \\
& \stackrel{(5)}{=}-b \tau(t)+\tilde{f}(t), \\
& =-b_{0} \tau(t)+\tilde{f}(t)+\tau(t)\left(b_{0}-b\right), \\
& =-b_{0} \tau(t)+f(t),
\end{aligned}
$$

where $b=1 / J_{1}$ is the input gain and $b_{0}$ is a design parameter, representing user's best knowledge of $b$. Term $f(t)$ is the resultant disturbance defined as: $f(t)=\frac{1}{J_{1}} \tau^{*}(t)+\tilde{d}^{*}(t)-\tilde{d}(t)+\tau(t)\left(b_{0}-b\right)+\varphi(t)$, which lumps the effects of neglected internal dynamics $\tilde{d}(t)$, unmodeled frictions, nonlinear dynamics, uncertainty in input gain selection $\tau(t)\left(b_{0}-b\right)$, and external disturbance $\varphi(t)$. It is also a function of unknown nominal control input $\tau^{*}(t)$, desired trajectory, and the desired nominal internal dynamic $\tilde{d}^{*}(t)$.

\subsection{Control rule}

In order to synthesize control rule in accordance to A4, methodology from [31,32] is used, in which a derivative feedback term $k_{2} \dot{e}_{\theta_{1}}$ is first added to both sides of the dynamic error Eq. (8), which gives:

$$
\ddot{e}_{\theta_{1}}+k_{2} \dot{e}_{\theta_{1}}=-b_{0} \tau(t)+k_{2} \dot{e}_{\theta_{1}}+f(t),
$$

leading to even simpler perturbed error-based model:

$$
\ddot{e}_{\theta_{1}}+k_{2} \dot{e}_{\theta_{1}}=-b_{0} \tau(t)+F(t)
$$

where $F(t)=k_{2} \dot{e}_{\theta_{1}}+f(t)$ is the system assumed total disturbance [12], to be estimated later using a specialized observer. Term $F(t)$ combines all the unknown/uncertain external and internal dynamics that affects the system output, including the term $k_{2} \dot{e}_{\theta_{1}}$, which consists the unknown target signal derivative. 
A following control action can be now proposed:

$$
\tau(t)=\left[u_{0}+\widehat{F}(t)\right] / b_{0},
$$

which avoids using (directly) reference time-derivatives, which is of great practical advantage. The outer-loop feedback controller $\left(u_{0}\right)$ for stabilizing the resultant dynamics (assuming proper disturbance rejection $\widehat{F}(t) \approx F(t)$ ), can be chosen to be just a static proportional action:

$$
u_{0}=k_{1} e_{\theta_{1}} .
$$

The application of (11) with (12) to system model (10) results in a following closed-loop error dynamics:

$$
\ddot{e}_{\theta_{1}}+k_{2} \dot{e}_{\theta_{1}}+k_{1} e_{\theta_{1}}=F(t)-\widehat{F}(t) \approx 0 \text {. }
$$

In order to get the controller coefficients $\left\{k_{1}, k_{2}\right\}>0$, a pole-placement method from [33] is used. The closed-loop tracking error characteristics polynomial can be matched with a Hurwitz polynomial: $s^{2}+k_{2} s+k_{1}=\left(s+\omega_{c}\right)^{2}$, from which the controller gains can be straightforwardly calculated as $k_{1}=\omega_{c}^{2}$ and $k_{2}=2 \omega_{c}$, where $\omega_{c}[\mathrm{rad} / \mathrm{s}]$ represents the desired, user-defined closed-loop bandwidth.

Remark 2. One can now clearly notice that the burden of delivering required control performance has been almost completely shifted onto an accurate and timely reconstruction of the total disturbance $F(t)$. This can be a determinative factor in selecting the proposed methodology to certain applications, as the target control system has to fulfill certain requirements related to minimum sampling time and maximum level of measurement noise.

\subsection{State and total disturbance reconstruction}

\subsubsection{Error-based RESO (proposed)}

Due to compliant nature of the considered torsional system, the undesired oscillations appear in the top two discs when the bottom one is moving relatively fast. In that case, both middle and top discs are generating a harmonic motion (disturbance) affecting bottom disc. Such periodic disturbance negatively effects the trajectory tracking performance, hence has to be mitigated by the proposed governing scheme, as stated in the control objective in Section 2.2.

Following the methodology presented in [27], the total disturbance can be treated as a sum of two signals $F(t)=F_{p}(t)+F_{w}(t)$, where $F_{p}=c_{0}+c_{1} t+c_{2} t^{2}+\cdots+c_{m-1} t^{(m-1)}$ is a polynomial disturbance approximation and $F_{w}(t)=a_{1} \sin \left(\omega_{r} t\right)+a_{2} \cos \left(\omega_{r} t\right)$ is a sinusoidal disturbance approximation. The harmonic disturbance $F_{w}(t)$ can be modeled as an unforced harmonic oscillator with resonant frequency $\omega_{\mathrm{r}}[\mathrm{rad} / \mathrm{s}]$, with its characteristic equation:

$$
\ddot{F}_{w}(t)+\omega_{r}^{2} F_{w}(t)=0
$$

Such sinusoidal disturbance model can now be included in the observer form by augmenting the system with two extra state variables: $x_{n+m+1}=F_{w}^{(m)}(t)$ and $x_{n+m+2}=F_{w}^{(m+1)}(t)$, where $m$ represents the number of extended states for $F_{p}$. One can thus rewrite (14) also as:

$$
F_{w}^{(m+2)}(t)+\omega_{r}^{2} F_{w}^{(m)}(t)=0 .
$$

In this particular work, one extended state variable $(m=1)$ is chosen to represent the polynomial disturbance $F_{p}(t)$. This, together with the baseline second order system dynamics $(n=2)$ and the above-mentioned harmonic disturbance $F_{w}(t)$ resulting in two extra states: $\dot{x}_{4}=x_{5}, \dot{x}_{5}=-\omega_{r}^{2} x_{4}$, makes the error dynamics (10) being expressed in state-space as:

$$
\begin{aligned}
\dot{\boldsymbol{x}}_{R} & =\boldsymbol{A}_{R} \boldsymbol{x}_{R}+\boldsymbol{b}_{R} \tau(t)+\boldsymbol{E}_{R} \dot{F}_{p}(t), \\
y_{R} & =\boldsymbol{C}_{R} \boldsymbol{x}_{R},
\end{aligned}
$$

where:

$$
\boldsymbol{x}_{R}=\left[\begin{array}{c}
x_{1} \\
x_{2} \\
x_{3} \\
x_{4} \\
x_{5}
\end{array}\right]=\left[\begin{array}{c}
e_{\theta_{1}} \\
\dot{e}_{\theta_{1}} \\
F(t) \\
\dot{F}_{w}(t) \\
\ddot{F}_{w}(t)
\end{array}\right], \quad \boldsymbol{E}_{R}=\left[\begin{array}{l}
0 \\
0 \\
1 \\
0 \\
0
\end{array}\right], \quad \boldsymbol{C}_{R}^{\top}=\left[\begin{array}{l}
1 \\
0 \\
0 \\
0 \\
0
\end{array}\right], \quad \boldsymbol{A}_{R}=\left[\begin{array}{ccccc}
0 & 1 & 0 & 0 & 0 \\
0 & -k_{2} & 1 & 0 & 0 \\
0 & 0 & 0 & 1 & 0 \\
0 & 0 & 0 & 0 & 1 \\
0 & 0 & 0 & -\omega_{r}^{2} & 0
\end{array}\right], \quad \boldsymbol{b}_{R}=\left[\begin{array}{c}
0 \\
-b_{0} \\
0 \\
0 \\
0
\end{array}\right] .
$$

For the above system, an error-based RESO is:

$$
\begin{aligned}
& \dot{\hat{\boldsymbol{x}}}_{R}=\boldsymbol{A}_{R} \hat{\boldsymbol{x}}_{R}+\boldsymbol{b}_{R} \tau(t)+\boldsymbol{L}_{R}\left(y_{R}-\hat{y}_{R}\right), \\
& \hat{y}_{R}=\boldsymbol{C}_{R} \hat{\boldsymbol{x}}_{R},
\end{aligned}
$$


where:

$$
\boldsymbol{L}_{R}=\left[\begin{array}{l}
\gamma_{1} \\
\gamma_{2} \\
\gamma_{3} \\
\gamma_{4} \\
\gamma_{5}
\end{array}\right], \quad \hat{\boldsymbol{x}}_{R}=\left[\begin{array}{c}
\hat{x}_{1} \\
\hat{x}_{2} \\
\hat{x}_{3} \\
\hat{x}_{4} \\
\hat{x}_{5}
\end{array}\right]=\left[\begin{array}{c}
\hat{e}_{\theta_{1}} \\
\hat{\hat{e}}_{\theta_{1}} \\
\widehat{F}(t) \\
\hat{\widehat{F}}_{w}(t) \\
\ddot{\widehat{F}}_{w}(t)
\end{array}\right] .
$$

One can notice that the state matrix $\boldsymbol{A}_{R}$ contains the design control coefficient $k_{2}$, which is embedded in the new observer. This is one of the key points of the proposed governing concept, which allows avoiding derivatives in the control rule (see A4), hence only a proportional feedback term can be used in (11). The derivative terms are conveniently reconstructed in the proposed framework by the means of the error-based observer. The observer state matrix $\boldsymbol{A}_{R}$ also contains an internal model of the harmonic dynamics with its resonant frequency $\omega_{r}$. For cases in which the frequency of harmonic disturbance $\omega_{r}$ is unknown or highly uncertain, specific method of obtaining it will be given later.

Now, similar to the controller tuning, a pole-placement technique can be used here to compute the observer gains. The estimation error state matrix is first given as:

$$
\boldsymbol{H}_{R}=\boldsymbol{A}_{R}-\boldsymbol{L}_{R} \boldsymbol{C}_{R}=\left[\begin{array}{ccccc}
-\gamma_{1} & 1 & 0 & 0 & 0 \\
-\gamma_{2} & -k_{2} & 1 & 0 & 0 \\
-\gamma_{3} & 0 & 0 & 1 & 0 \\
-\gamma_{4} & 0 & 0 & 0 & 1 \\
-\gamma_{4} & 0 & 0 & -\omega_{r}^{2} & 0
\end{array}\right]
$$

and the characteristic estimation error polynomial is:

$$
\begin{aligned}
& P_{r}(s)=\operatorname{det}\left(s \boldsymbol{I}-\boldsymbol{H}_{R}\right), \\
& P_{r}(s)=s^{5}+\left(\gamma_{1}+k_{2}\right) s^{4}+\left(w_{2}^{2}+\gamma_{2}+\gamma_{1} k_{2}\right) s^{3}+\left(\gamma_{3}+k_{2} w_{r}^{2}+\gamma_{1} w_{r}^{2}\right) s^{2}+\left(\gamma_{4}+\gamma_{2} w_{r}^{2}+\gamma_{1} k_{2} w_{r}^{2}\right) s+\left(\gamma_{3} w_{r}^{2}+\gamma_{5}\right) .
\end{aligned}
$$

The goal is to place the roots of a characteristic polynomial in the left-hand side of the complex plane. Matching the characteristic polynomial $P_{r}(s)$ with a Hurwitz polynomial $P_{r}(s)=\left(s+\omega_{0}\right)^{5}$ gives a following new set of observer gains: $\gamma_{1}=5 \omega_{o}-k_{2}, \gamma_{2}=10 \omega_{o}^{2}-\omega_{r}^{2}-k_{2} \gamma_{1}, \gamma_{3}=10 \omega_{o}^{3}-\gamma_{1} \omega_{r}^{2}-k_{2} \omega_{r}^{2}, \gamma_{4}=5 \omega_{o}^{4}-\gamma_{2} \omega_{r}^{2}-k_{2} \gamma_{1} \omega_{r}^{2}, \gamma_{5}=\omega_{o}^{5}-\gamma_{3} \omega_{r}^{2}$.

An illustrative depiction of the proposed error-based ADRC solution is presented in Fig. 3 and its comprehensive stability proof is given in the next section.

\subsubsection{Error-based GPIO (conventional, for comparison)}

A design of a generalized proportional integral observer (GPIO) is also shown here for the considered uncertain system (10), as it will be later used in a comparison study. The class of GPIO-based controllers is considered to be standard solution for cases in which the system is subjected to complex disturbances [15,34,35]. The GPIO utilizes solely a polynomial-type representation of lumped uncertainty, meaning the more complex the overall disturbance is, the more extended state variables, representing higher orders of a polynomial signal, are introduced to represent its behavior accurately. Comparing it with the total disturbance $F(t)$ defined previously for the error-based RESO, it is similar to considering just the $F_{p}(t)$ part of certain order.

In this work, the considered system (10) is artificially augmented with three extra state variables $(m=3)$, representing the lumped uncertainty, ${ }^{3}$ to match its order with the order of the proposed error-based RESO (for the purpose of upcoming comparison). The extended plant model for the GPIO design purpose is thus expressed as:

$$
\begin{aligned}
\dot{\boldsymbol{x}}_{G} & =\boldsymbol{A}_{G} \boldsymbol{x}_{G}+\boldsymbol{b}_{G} \tau(t)+\boldsymbol{E}_{G} F^{(3)}(t), \\
y_{G} & =\boldsymbol{C}_{G} \boldsymbol{x}_{G},
\end{aligned}
$$

with:

$$
\boldsymbol{x}_{G}=\left[\begin{array}{c}
x_{1} \\
x_{2} \\
x_{3} \\
x_{4} \\
x_{5}
\end{array}\right]=\left[\begin{array}{c}
e_{\theta_{1}} \\
\dot{e}_{\theta_{1}} \\
F(t) \\
\dot{F}(t) \\
\ddot{F}(t)
\end{array}\right], \quad \boldsymbol{E}_{G}=\left[\begin{array}{l}
0 \\
0 \\
0 \\
0 \\
1
\end{array}\right], \quad \boldsymbol{C}_{G}^{\top}=\left[\begin{array}{l}
1 \\
0 \\
0 \\
0 \\
0
\end{array}\right], \quad \boldsymbol{A}_{G}=\left[\begin{array}{ccccc}
0 & 1 & 0 & 0 & 0 \\
0 & -k_{2} & 1 & 0 & 0 \\
0 & 0 & 0 & 1 & 0 \\
0 & 0 & 0 & 0 & 1 \\
0 & 0 & 0 & 0 & 0
\end{array}\right], \quad \boldsymbol{b}_{G}=\left[\begin{array}{c}
0 \\
-b_{0} \\
0 \\
0 \\
0
\end{array}\right],
$$

\footnotetext{
${ }^{3}$ It is assumed that $F(t)$ has higher-order dynamics, which seems reasonable given the oscillatory influence of the underactuated dynamics.
} 


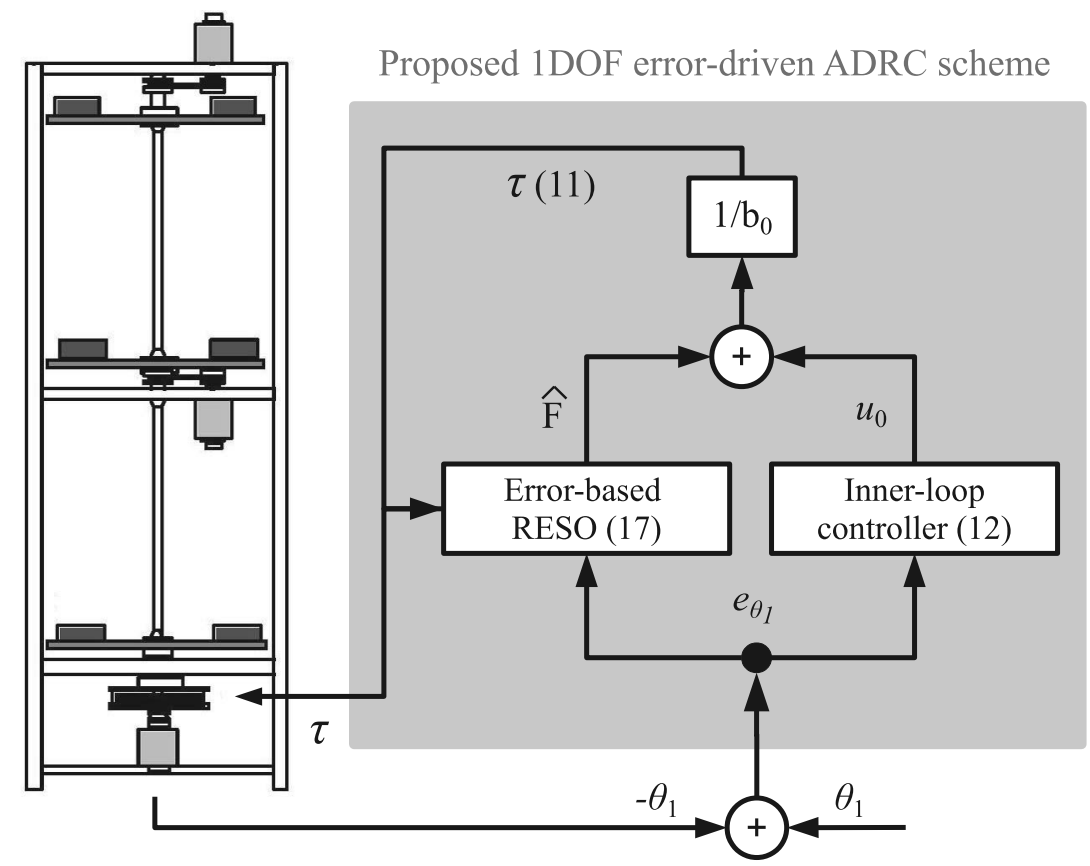

Fig. 3. Proposed custom ADRC scheme, in which RESO and feedback controller are merged into a 1DOF structure expressed in error-based form.

where term $F(t)$ is assumed to be a smooth function with bounded time derivatives. For the above system, a conventional GPIO in error domain is proposed as:

$$
\begin{aligned}
& \dot{\hat{\boldsymbol{x}}}_{G}=\boldsymbol{A}_{G} \hat{\boldsymbol{x}}_{G}+\boldsymbol{b}_{G} \tau(t)+\boldsymbol{L}_{G}\left(y_{G}-\hat{y}_{G}\right), \\
& \hat{y}_{G}=\boldsymbol{C}_{G} \hat{\boldsymbol{x}}_{G},
\end{aligned}
$$

where:

$$
\boldsymbol{L}_{G}=\left[\begin{array}{l}
l_{1} \\
l_{2} \\
l_{3} \\
l_{4} \\
l_{5}
\end{array}\right], \hat{\boldsymbol{x}}_{G}=\left[\begin{array}{c}
\hat{x}_{1} \\
\hat{x}_{2} \\
\hat{x}_{3} \\
\hat{x}_{4} \\
\hat{x}_{5}
\end{array}\right]^{T}=\left[\begin{array}{c}
\hat{e}_{\theta_{1}} \\
\dot{\hat{e}}_{\theta_{1}} \\
\widehat{F}(t) \\
\hat{\hat{F}}(t) \\
\ddot{\hat{F}}(t)
\end{array}\right]^{T} .
$$

For consistency, same tuning is used as for the error-based RESO. Here, however, the estimation-error state matrix has the form:

$$
\boldsymbol{H}_{G}=\boldsymbol{A}_{G}-\boldsymbol{L}_{G} \boldsymbol{C}_{G}=\left[\begin{array}{ccccc}
-l_{1} & 1 & 0 & 0 & 0 \\
-l_{2} & -k_{2} & 1 & 0 & 0 \\
-l_{3} & 0 & 0 & 1 & 0 \\
-l_{4} & 0 & 0 & 0 & 1 \\
-l_{4} & 0 & 0 & 0 & 0
\end{array}\right],
$$

and the characteristic estimation error polynomial is:

$$
\begin{aligned}
& P_{o}(s)=\operatorname{det}\left(s \boldsymbol{I}-\boldsymbol{H}_{G}\right), \\
& P_{o}(s)=s^{5}+\left(k_{2}+l_{1}\right) s^{4}+\left(l_{2}+k_{2} l_{1}\right) s^{3}+l_{3} s^{2}+l_{4} s+l_{5} .
\end{aligned}
$$

which results in a following set of GPIO gains:

$$
\begin{aligned}
& l_{1}=5 \omega_{0}-k_{2}, l_{2}=10 \omega_{o}^{2}-k_{2} l_{1}, \\
& l_{3}=10 \omega_{0}^{3}, l_{4}=5 \omega_{0}^{4}, l_{5}=\omega_{0}^{5} .
\end{aligned}
$$




\section{Stability analysis}

The theoretical investigation of the generalized version of the proposed error-based ADRC with RESO is presented next and its analysis is based on singular perturbation theory [36-38]. Compared to [36], here the stability proof will not be divided explicitly into slow and fast dynamics part, representing the controller and observer loops, respectively, since in the considered error-based ADRC these two loops are incorporated into a single closed-loop error dynamics.

First, a general expression combining observer and controller parts can be written as:

$$
\begin{aligned}
\dot{\boldsymbol{q}} & =\left(\boldsymbol{A}-\boldsymbol{l c}^{\top}\right) \boldsymbol{q}+\boldsymbol{h} \eta, \eta=\dot{F} \\
\boldsymbol{A} & =\left[\begin{array}{ccccc}
0 & 1 & 0 & 0 & 0 \\
-k_{1} & -k_{2} & 1 & 0 & 0 \\
0 & 0 & 0 & 1 & 0 \\
0 & 0 & 0 & 0 & 1 \\
0 & 0 & 0 & -\omega_{r}^{2} & 0
\end{array}\right], \quad \boldsymbol{h}=\left[\begin{array}{l}
0 \\
0 \\
1 \\
0 \\
0
\end{array}\right], \quad \boldsymbol{c}=\left[\begin{array}{l}
1 \\
0 \\
0 \\
0 \\
0
\end{array}\right] .
\end{aligned}
$$

with $\boldsymbol{q}^{\top}=\left[q_{1} q_{2} q_{3} q_{4} q_{5}\right]=\left[\left(e_{\theta_{1}}-\hat{e}_{\theta_{1}}\right)\left(\dot{e}_{\theta_{1}}-\hat{\dot{e}}_{\theta_{1}}\right)(F-\widehat{F})\left(\dot{F}_{w}-\hat{\dot{F}}_{w}\right)\left(\ddot{F}_{w}-\hat{\vec{F}}_{w}\right)\right]$ being the state vector of new system coordinates, $\boldsymbol{I}^{\top}=\left[\begin{array}{llll}\gamma_{1} & \gamma_{2} & \ldots & \gamma_{5}\end{array}\right]$ being vector of observer gains, and $k_{1}$ and $k_{2}$ being the proportional and derivative controller gain, respectively.

Next, calling both the estimation error matrix $\boldsymbol{H}=\boldsymbol{A}-\boldsymbol{l c}^{\top}$ and the previously used pole-placement-based observer tuning [33], one can induce, by analyzing their forms for different observer orders, that:

$$
\begin{aligned}
\operatorname{det}(\lambda \boldsymbol{I}-\boldsymbol{H}) & =\lambda^{5}+s_{1} \lambda^{4}+s_{2} \lambda^{3}+s_{3} \lambda^{2}+s_{4} \lambda+s_{5}, \\
& =\left(\lambda+\omega_{0}\right)^{5},
\end{aligned}
$$

for $\left[s_{1}, \ldots, s_{5}\right]=\left[\beta_{1} \omega_{0}, \ldots, \beta_{5} \omega_{0}^{5}\right]$. The above yields:

$$
\lambda_{m}\{\boldsymbol{H}\}=-\omega_{0}, \quad \forall m=1, \ldots, 5 .
$$

The coefficients polynomial (25) are thus computed as:

$$
\begin{aligned}
& s_{1}=\overbrace{k_{2}}^{r_{1}}+l_{1}=\beta_{1} \omega_{0} \Rightarrow l_{1}=\beta_{1} \omega_{0}-r_{1}, \\
& s_{2}=\overbrace{\omega_{r}^{2}+k_{2} l_{1}}^{r_{2}}+k_{1}+l_{2}=\beta_{2} \omega_{o}^{2} \Rightarrow l_{2}+k_{1}=\beta_{2} \omega_{o}^{2}-r_{2}, \\
& s_{3}=\overbrace{\omega_{r}^{2} l_{1}+\omega_{r}^{2} k_{2}}^{r_{3}}+l_{3}=\beta_{3} \omega_{0}^{3} \Rightarrow l_{3}=\beta_{3} \omega_{0}^{3}-r_{3} \\
& s_{4}=\overbrace{\omega_{r}^{2} l_{2}+\omega_{r}^{2} k_{2} l_{1}+k_{1} \omega_{r}^{2}}^{r_{4}}+l_{4}=\beta_{4} \omega_{o}^{4} \Rightarrow l_{4}=\beta_{4} \omega_{o}^{4}-r_{4}, \\
& s_{5}=\overbrace{l_{3} \omega_{r}^{2}}^{r_{5}}+l_{5}=\beta_{5} \omega_{0}^{5} \Rightarrow l_{5}=\beta_{5} \omega_{0}^{5}-r_{5},
\end{aligned}
$$

where $r_{i}$ are uniquely defined by $k_{i}$ and $\omega_{r}^{2}$. This further allows the estimation error state matrix $\boldsymbol{H}$ to be rewritten as:

$$
\begin{aligned}
\boldsymbol{H} & =\left[\begin{array}{ccccc}
-\gamma_{1} & 1 & 0 & 0 & 0 \\
-\gamma_{2}-k_{1} & -k_{2} & 1 & 0 & 0 \\
-\gamma_{3} & 0 & 0 & 1 & 0 \\
-\gamma_{4} & 0 & 0 & 0 & 1 \\
-\gamma_{5} & 0 & 0 & -\omega_{r}^{2} & 0
\end{array}\right] \\
& =\left[\begin{array}{ccccc}
-\beta_{1} \omega_{0} & 1 & 0 & 0 & 0 \\
-\beta_{2} \omega_{0}^{2} & 0 & 1 & 0 & 0 \\
-\beta_{3} \omega_{0}^{3} & 0 & 0 & 1 & 0 \\
-\beta_{4} \omega_{0}^{4} & 0 & 0 & 0 & 1 \\
-\beta_{5} \omega_{0}^{5} & 0 & 0 & 0 & 0
\end{array}\right]+\left[\begin{array}{ccccc}
r_{1} & 1 & 0 & 0 & 0 \\
r_{2} & -k_{2} & 1 & 0 & 0 \\
r_{3} & 0 & 0 & 1 & 0 \\
r_{4} & 0 & 0 & 0 & 1 \\
r_{5} & 0 & 0 & -\omega_{r}^{2} & 0
\end{array}\right]
\end{aligned}
$$


Now, for the system given in (24), a following change of coordinates is introduced: $q_{1}=\omega_{0}^{-4} \xi_{1}, q_{2}=\omega_{0}^{-3} \xi_{2}, \ldots, q_{5}=\xi_{5}$, with its general form $q_{i}=\omega_{o}^{i-3} \xi_{i}$, for $i=1, \ldots, 5$, or expressed alternatively in matrix representation as $\boldsymbol{q}=\boldsymbol{\Lambda} \xi$, with $\boldsymbol{\Lambda}=\operatorname{diag}\left[\omega_{0}^{-4} \omega_{0}^{-3} \cdots \omega_{0}^{-1} 1\right]$ and $\xi=\left[\xi_{1} \xi_{2} \cdots \xi_{4} \xi_{5}\right]$. This allows to rewrite (24) as:

$$
\boldsymbol{\Lambda} \dot{\xi}=\left(\boldsymbol{A}-\boldsymbol{l c}^{\top}\right) \boldsymbol{\Lambda} \boldsymbol{\xi}+\boldsymbol{h} \eta=\boldsymbol{H} \boldsymbol{\Lambda} \xi+\boldsymbol{h} \eta
$$

which yields:

$$
\frac{1}{\omega_{0}} \dot{\boldsymbol{\xi}}=\boldsymbol{H}_{q} \boldsymbol{\xi}+\frac{1}{\omega_{0}} \boldsymbol{h} \eta \Rightarrow \varepsilon \dot{\boldsymbol{\xi}}=\boldsymbol{H}_{q} \boldsymbol{\xi}+\varepsilon \boldsymbol{h} \eta
$$

with $\varepsilon=\frac{1}{\omega_{o}}$, and $\boldsymbol{H}_{q}=\boldsymbol{A}_{q}+\varepsilon \boldsymbol{H}_{\varepsilon}$, where:

$$
\begin{aligned}
\boldsymbol{A}_{q} & =\left[\begin{array}{ccccc}
-\beta_{1} & 1 & 0 & 0 & 0 \\
-\beta_{2} & 0 & 1 & 0 & 0 \\
-\beta_{3} & 0 & 0 & 1 & 0 \\
-\beta_{4} & 0 & 0 & 0 & 1 \\
-\beta_{5} & 0 & 0 & 0 & 0
\end{array}\right], \\
\boldsymbol{H}_{\varepsilon} & =\left[\begin{array}{ccccc}
r_{1} & 0 & 0 & 0 & 0 \\
\varepsilon\left(r_{2}-k_{1}\right) & -\varepsilon^{n-2} k_{2} & 0 & 0 & 0 \\
\varepsilon^{2} r_{3} & 0 & 0 & 0 & 0 \\
\varepsilon^{3} r_{4} & 0 & 0 & 0 & 0 \\
\varepsilon^{4} r_{5} & 0 & 0 & -\varepsilon \omega_{r}^{2} & 0
\end{array}\right],
\end{aligned}
$$

with $\omega_{0}, \omega_{r}, \beta_{i}$ 's, and $k_{i}$ 's being completely determined in (28).

A proper stability analysis of the general error-based ADRC with RESO can now be performed. Let us consider an observer error dynamics (31) with its initial condition:

$$
\varepsilon \dot{\xi}=\boldsymbol{H}_{q} \xi+\varepsilon h \cdot \eta, \xi(0)=\xi_{0}
$$

It is an initial value problem of a standard singularly perturbed linear system of differential equations with a small positive parameter $\varepsilon=\frac{1}{\omega_{0}}$, that depends on the observer bandwidth $\omega_{0}$. Note that matrix $\boldsymbol{H}_{q}$ and the estimation-error state matrix $\boldsymbol{H}$ are similar $\left(\boldsymbol{H}=\boldsymbol{\Lambda} \boldsymbol{H}_{\boldsymbol{q}} \boldsymbol{\Lambda}^{-1}\right.$ with $\left.H_{q}=A_{q}+\epsilon H_{\epsilon}\right)$ and have the same eigenvalues. Thus, $\lambda_{i}\left(\boldsymbol{H}_{q}\right)=\lambda_{i}(\boldsymbol{H})=-\omega_{o}$ and $\lambda_{i}\left(\boldsymbol{A}_{q}\right)=-1, \forall i=1, \ldots, 5$, which implies $\boldsymbol{H}_{q}$ and $\boldsymbol{A}_{q}$ are Hurwitz. This implies that $\omega_{\mathrm{r}}$ in state matrix $\boldsymbol{A}$ does not affect the stability of the coefficient matrix $\boldsymbol{H}_{q}$ in (34) as long as $\omega_{r}$ is finite. As in [36], it can be thus concluded that for the existing of the derivative of total disturbance $\dot{F}$, if $\left\|\xi_{0}\right\|=O(\varepsilon)$ i.e. $\xi_{0}$ lies in its stable initial manifold, there will be no danger of large magnitude transients in system (34) as $\varepsilon \rightarrow 0$ for $t>0$, and the system is exponentially stable and uniformly asymptotically stable (for stability within the stable initial manifold for $\xi_{0}$, it is not the stability in the Lyapunov sense). On the other hand, it is easy to verify that $\boldsymbol{H}_{q}$ is Hurwitz for any finite $\omega_{r}$ in $\boldsymbol{H}_{q}$. This implies that $\omega_{\mathrm{r}}$ in state matrix $\boldsymbol{A}(24)$ does not affect the stability of the coefficient matrix $\boldsymbol{H}_{q}$ as long as its value is finite. In the spirit of Theorems 3.1 and 4.1 of [36], main stability theorem is presented as shown below.

Theorem 1. If $\left\|\xi_{0}\right\|=O(\varepsilon)$ and $\eta=\dot{F}$ exists, then:

(i) the asymptotic solution of (34),

$\varepsilon \dot{\xi}=\boldsymbol{H}_{q} \xi+\varepsilon h \cdot \eta=\left(\boldsymbol{A}_{q}+\varepsilon \boldsymbol{H}_{\varepsilon}\right) \xi+\varepsilon h \cdot \eta, \xi(0)=\xi_{0}$,

is uniformly valid for all finite time $L$ with $0 \leqslant t \leqslant L<\infty$, and it has the following form:

$$
\xi(\varepsilon, t)=\exp \left(\boldsymbol{A}_{q} \frac{t}{\varepsilon}\right) \xi_{0}+O\left(\varepsilon^{2}\right)+\varepsilon\left[\boldsymbol{A}_{q}^{-1} h \cdot \eta+\exp \left(\boldsymbol{A}_{q} \frac{t}{\varepsilon}\right)\left\{\boldsymbol{A}_{q}^{-1} h \cdot \eta(0)\right\}-\boldsymbol{H}_{0} \int_{0}^{t} \exp \left[\boldsymbol{A}_{q}\left(\frac{t-s}{\varepsilon}\right)\right] \boldsymbol{A}_{q}^{-1} h \cdot \eta(s) d s\right] ;
$$

(ii) there exists an $\varepsilon^{*}>0$ such that for all $\varepsilon \in\left[0, \varepsilon^{*}\right]$ the leading term of the solution $\xi(\varepsilon, t)$ in the initial layer of system (34) is exponentially stable;

(iii) for all $\xi \in \Omega=\left[-\rho_{1}, \rho_{2}\right]^{5} \subset \mathbb{R}^{5}$ with $\rho_{2}=\gamma \max \left\{\omega_{c}, \frac{1}{\omega_{o}},\left\|\boldsymbol{H}_{q}\right\|\right\}$ for some constant $\gamma>0$, then there exists positive constant $C_{2}$, independent of $\varepsilon$, and the solution of (34) satisfies:

$$
\begin{aligned}
& \|\xi(\varepsilon, t)\| \leqslant \varepsilon C_{1} \exp \left[-\left(\frac{1}{2}-\varepsilon C_{1} C_{2}\right) \frac{t}{\varepsilon}\right], \\
& \|\eta\| \cdot\|\xi(\varepsilon, t)\|^{-1} \leqslant C_{2}
\end{aligned}
$$

where $C_{1}=\sqrt{5}+\frac{1}{\omega_{c}} \sum_{j=1}^{4} \frac{L^{j}}{j !}\left\|\left(\boldsymbol{H}_{q}+\mathbf{I}_{5}\right)^{j^{2}}\right\|$ with $\mathbf{I}_{5}$ is the fifth order identity matrix and: 


$$
\boldsymbol{H}_{0}=\left[\begin{array}{ccccc}
2 \omega_{c} & 0 & 0 & 0 & 0 \\
0 & -2 \omega_{c} & 0 & 0 & 0 \\
0 & 0 & 0 & 0 & 0 \\
0 & 0 & 0 & 0 & 0 \\
0 & 0 & 0 & 0 & 0
\end{array}\right]
$$

Also, following remarks can be made.

- One can see that from (i) of Theorem 1 and the classic result of the singular perturbation theory, (ii) holds if (i) of Theorem 1 holds.

- Theorem 1 states that the system (34) is exponentially stable and uniformly asymptotically stable if $\left\|\xi_{0}\right\|=O(\varepsilon)=O\left(\frac{1}{\omega_{0}}\right)$ and $\eta=\dot{F}$ exists (the result holds, for stability within the stable initial manifold for $\xi_{0}$, it is not the stability in the Lyapunov sense). This means that the stability results in [36] can be extended to the proposed error-based ADRC with RESO, which the error dynamic system (34) has a more general coefficient matrix $\boldsymbol{H}_{q}=\boldsymbol{A}_{q}+\varepsilon \boldsymbol{H}_{\varepsilon}$.

- The restriction of the condition of the existing of $\dot{F}$ could be relaxed. If we allow the total disturbance $F$ to belong to some larger class of functions, for example, $F$ is a function of Sobolev class $W_{\text {loc }}^{1,1}$ and it is differentiable a.e. ${ }^{4}$, then its weak derivative is represented by the point-wise derivative (for details see [39]), thus all the results in Theorem 1 hold.

\section{Frequency analysis}

The performance of the proposed RESO-based control algorithm is compared here with conventional GPIO-based approach through numerical simulations in frequency domain. The considered system is presented in the transfer function form (see Fig. 4), with $\theta_{1}^{*}(s), \theta_{1}(s), E_{\theta_{1}}(s), \tau(s), N(s)$, and $\tau_{l t}(s)$ representing the reference signal, system output, tracking error, control input, measurement noise, and external disturbance (load torque), respectively.

The plant transfer function $G_{p}(s)$ is derived system using the following relations between the input torque and the angular positions of corresponding discs:

$$
\frac{\theta_{1}(s)}{\tau(s)}=\frac{N_{1}(s)}{D(s)}, \frac{\theta_{2}(s)}{\tau(s)}=\frac{N_{2}(s)}{D(s)}, \frac{\theta_{3}(s)}{\tau(s)}=\frac{N_{3}(s)}{D(s)},
$$

for $N_{1}(s)=a_{11} s^{4}+a_{12} s^{3}+a_{13} s^{2}+a_{14} s+a_{15}, N_{2}(s)=a_{21} s^{2}+a_{22} s+a_{23}, N_{3}(s)=a_{31}$, and $D(s)=a_{41} s^{6}+a_{42} s^{5}+a_{43} s^{4}+a_{44} s^{3}+$ $a_{45} s^{2}+a_{46}$, with specific values of coefficients given in the Appendix A.

The transfer function of the proposed error-based ADRC with RESO given as:

$$
G_{c}(s)=\frac{g_{11} s^{5}+g_{12} s^{4}+g_{13} s^{3}+g_{14} s^{2}+g_{15} s+g_{16}}{b_{0}\left(s^{2}+\omega_{r}\right)\left[s^{2}+\left(k_{2}+\gamma_{1}\right) s+\gamma_{2}+\gamma_{1} k_{2}\right]},
$$

is obtained from (12) and (16) using Laplace transforms, with coefficients: $g_{11}=k_{1}, g_{12}=k_{1} \gamma_{1}+k_{1} k_{2}, g_{13}=k_{1} \gamma_{2}+k_{1} k_{2} \gamma_{1}+$ $k_{2} \gamma_{3}+\gamma_{4}+\omega_{r}^{2} k_{1}, g_{14}=k_{1} \beta_{3}+k_{2} \beta_{4}+\beta_{5}+\omega_{r}^{2} g_{12}, g_{15}=k_{1} \gamma_{4}+k_{2} \gamma_{5}+\omega_{r}^{2}\left(k_{1} \gamma_{2}+k_{1} k_{2} \gamma_{1}+k_{2} \gamma_{3}\right)$ and $g_{16}=k_{1} \gamma_{5}+k_{1} \gamma_{3} \omega_{r}^{2}$. On the other hand, the transfer function of the error-based ADRC with conventional GPIO is derived according to (12) and (19) by substituting $\omega_{r}=0$ and $\gamma_{i}=l_{i}$ for $i=1,2,3,4,5$ in (24).

In order to analyze the system robustness under different control frameworks, stability margins, including positive gain margin (+GM), negative gain margin (-GM), and phase margin (PM), are calculated based on Bode diagrams [40] of a loop transfer function $W(s)=G_{c}(s) G_{p}(s)$. The analysis is performed for different values of tuning parameters $\left(\omega_{c}, \omega_{0}, \omega_{r}\right)$ and the obtained comparison results between the proposed RESO- and the standard GPIO-based control systems are gathered in Table 1 (for $\omega_{c}=10 \mathrm{rad} / \mathrm{s}$ ) and in Table 2 (for $\omega_{c}=20 \mathrm{rad} / \mathrm{s}$ ). It can be noted that, for low values of RESO parameter $\omega_{r}$, both control algorithms have similar stability margins, and increasing $\omega_{r}$ provides better robustness in RESO-based control than in GPIO-based control. Also, increasing the observer bandwidth $\omega_{0}$ increases the robustness of the overall system.

To evaluate tracking and external disturbance rejection performance, the transfer function from reference $\theta_{1}^{*}(s)$ to tracking error $E_{\theta_{1}}(s)$ :

$$
G_{e}(s)=\frac{E_{\theta_{1}}(s)}{\theta_{1}^{*}(s)}=1-\frac{G_{c}(s) G_{p}(s)}{1+G_{c}(s) G_{p}(s)},
$$

and from external disturbance $\tau_{l} t(s)$ to output $\theta_{1}(s)$ :

$$
G_{d}(s)=\frac{\theta_{1}(s)}{\tau_{l t}(s)}=\frac{G_{p}(s)}{1+G_{c}(s) G_{p}(s)}
$$

\footnotetext{
${ }^{4}$ Definition. An almost everywhere differentiable function $g$ is a function that is differentiable except on a set of measure zero. That is, $g$ exists point-wise a.e.
} 


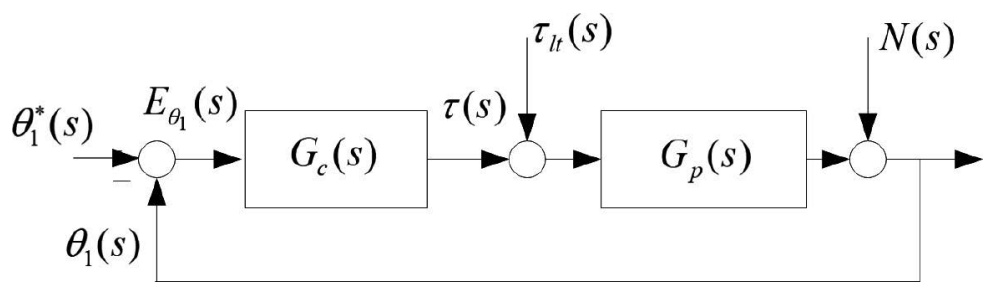

Fig. 4. Transfer function form of the considered control system.

Table 1

The systems robustness indices for $\omega_{c}=10 \mathrm{rad} / \mathrm{s}$.

\begin{tabular}{|c|c|c|c|c|c|c|c|c|c|c|}
\hline & \multirow{2}{*}{$\begin{array}{l}\omega_{o} \\
\omega_{r}\end{array}$} & \multicolumn{3}{|c|}{$8 \omega_{c}$} & \multicolumn{3}{|c|}{$16 \omega_{c}$} & \multicolumn{3}{|c|}{$24 \omega_{c}$} \\
\hline & & $4 \mathrm{rad} / \mathrm{s}$ & $16 \mathrm{rad} / \mathrm{s}$ & $32 \mathrm{rad} / \mathrm{s}$ & $4 \mathrm{rad} / \mathrm{s}$ & $16 \mathrm{rad} / \mathrm{s}$ & $32 \mathrm{rad} / \mathrm{s}$ & $4 \mathrm{rad} / \mathrm{s}$ & $16 \mathrm{rad} / \mathrm{s}$ & $32 \mathrm{rad} / \mathrm{s}$ \\
\hline GPIO & $\begin{array}{c}+\mathrm{GM}[\mathrm{dB}] \\
-\mathrm{GM}[\mathrm{dB}] \\
\left.\operatorname{PM}^{\circ}\right]\end{array}$ & & $\begin{array}{c}9.96 \\
-6.18 \\
18.6\end{array}$ & & & $\begin{array}{c}10.8 \\
-8.82 \\
25\end{array}$ & & & $\begin{array}{c}11.1 \\
-9.32 \\
27.2\end{array}$ & \\
\hline RESO & $\begin{array}{c}+\mathrm{GM}[\mathrm{dB}] \\
-\mathrm{GM}[\mathrm{dB}] \\
\operatorname{PM}\left[^{\circ}\right]\end{array}$ & $\begin{array}{l}9.97 \\
-6.2 \\
18.7\end{array}$ & $\begin{array}{c}10 \\
-6.64 \\
18.9\end{array}$ & $\begin{array}{c}10.2 \\
-7.28 \\
19.9\end{array}$ & $\begin{array}{c}-8.82 \\
10.8 \\
25\end{array}$ & $\begin{array}{c}10.9 \\
-8.89 \\
25\end{array}$ & $\begin{array}{c}10.9 \\
-9.13 \\
25.3\end{array}$ & $\begin{array}{c}11.1 \\
-9.32 \\
27.2\end{array}$ & $\begin{array}{c}11.1 \\
-9.35 \\
27.3\end{array}$ & $\begin{array}{l}11.2 \\
-9.7 \\
27.4\end{array}$ \\
\hline
\end{tabular}

Table 2

The systems robustness indices for $\omega_{c}=20 \mathrm{rad} / \mathrm{s}$.

\begin{tabular}{|c|c|c|c|c|c|c|c|c|c|c|}
\hline & \multirow{2}{*}{$\begin{array}{l}\omega_{o} \\
\omega_{r}\end{array}$} & \multicolumn{3}{|c|}{$8 \omega_{c}$} & \multicolumn{3}{|c|}{$16 \omega_{c}$} & \multicolumn{3}{|c|}{$24 \omega_{c}$} \\
\hline & & $4 \mathrm{rad} / \mathrm{s}$ & $16 \mathrm{rad} / \mathrm{s}$ & $32 \mathrm{rad} / \mathrm{s}$ & $4 \mathrm{rad} / \mathrm{s}$ & $16 \mathrm{rad} / \mathrm{s}$ & $32 \mathrm{rad} / \mathrm{s}$ & $4 \mathrm{rad} / \mathrm{s}$ & $16 \mathrm{rad} / \mathrm{s}$ & $32 \mathrm{rad} / \mathrm{s}$ \\
\hline GPIO & $\begin{array}{c}+\mathrm{GM}[\mathrm{dB}] \\
-\mathrm{GM}[\mathrm{dB}] \\
\mathrm{PM}\left[^{\circ}\right]\end{array}$ & & $\begin{array}{c}10 \\
-6.27 \\
18\end{array}$ & & & $\begin{array}{c}10.8 \\
-8.16 \\
24.1\end{array}$ & & & $\begin{array}{c}11.1 \\
-9.02 \\
27.2\end{array}$ & \\
\hline RESO & $\begin{array}{c}+\mathrm{GM}[\mathrm{dB}] \\
-\mathrm{GM}[\mathrm{dB}] \\
\mathrm{PM}\left[^{\circ}\right]\end{array}$ & $\begin{array}{c}10 \\
-6.28 \\
18.1\end{array}$ & $\begin{array}{c}10.1 \\
-6.48 \\
18.4\end{array}$ & $\begin{array}{c}10.3 \\
-7.19 \\
19.4\end{array}$ & $\begin{array}{c}10.8 \\
-8.17 \\
24.8\end{array}$ & $\begin{array}{c}10.9 \\
-8.23 \\
24.9\end{array}$ & $\begin{array}{c}10.9 \\
-8.46 \\
25.1\end{array}$ & $\begin{array}{c}11.1 \\
-9.02 \\
27.2\end{array}$ & $\begin{array}{c}11.1 \\
-9.03 \\
27.2\end{array}$ & $\begin{array}{c}11.1 \\
-9.19 \\
27.3\end{array}$ \\
\hline
\end{tabular}

are derived and their magnitude frequency responses are shown in Figs. 5 and 6, respectively. The results are presented for different $\omega_{c}$ and $\omega_{r}$ but with a fixed $\omega_{0}=16 \omega_{c}$. From the figures, one can notice that if the reference signal and/or load torque have the same tuned resonant frequency $\left(\omega_{r}=8 \mathrm{rad} / \mathrm{s}\right.$ and $\left.\omega_{r}=16 \mathrm{rad} / \mathrm{s}\right)$, the RESO-based control systems achieves practically ideal reference tracking and disturbance rejection. ${ }^{5}$ Moreover, it is evident that for the same controller bandwidth $\omega_{c}$, RESO-based control provides better tracking performance and disturbance rejection performance than the GPIO-based scheme in the relative wide frequency ranges $\Delta \omega_{1}$ (for $\omega_{c}=10 \mathrm{rad} / \mathrm{s}$ ) and $\Delta \omega_{2}$ (for $\omega_{c}=20 \mathrm{rad} / \mathrm{s}$ ). Also, one can see that the control performance for lower frequency can be improved by increasing $\omega_{c}$ and, as expected, the GPIO-based control enables better performance in that frequency range.

Regarding the measurement noise sensitivity, it is analyzed based on frequency response of the transfer function from the measurement noise $N(s)$ to the control signal $\tau(s)$ :

$$
G_{n}(s)=\frac{\tau(s)}{N(s)}=\frac{G_{c}(s)}{1+G_{c}(s) G_{p}(s)} .
$$

The frequency responses of (42) for GPIO- and RESO-based control methods with different tuned parameters $\omega_{c}$ and $\omega_{0}$ are presented in Fig. 7. It should be pointed out that the measurement noise is mainly concentrated at high frequencies and thus the behavior of the $G_{n}(s)$ at high frequencies is of primary importance. From Fig. 7, one can see that for the same value of tuned parameters $\omega_{c}$ and $\omega_{0}$, both control algorithms have practically identical robustness to measurement noise. Consequently, it means that tuning of parameter $\omega_{r}$ does not have effect on the measurement noise sensitivity, but increasing $\omega_{c}$ and $\omega_{o}$ reduces the measurement noise robustness. Therefore, it should be noted that tuning of $\omega_{c}$ and $\omega_{0}$ in practice should be a trade-off between tracking performance, robustness indices, and measurement noise sensitivity.

Remark 3. The straightforwardness and application simplicity of the pole-placement tuning methodology in ADRC made it a practically appealing approach, which has been successfully transitioned into industry and now can be found, for example, in embedded chips from Texas Instruments called InstaSPIN.

\footnotetext{
${ }^{5}$ The magnitude frequency response of $G_{e}(s)$ and $G_{d}(s)$ is less than $-160 \mathrm{~dB}$, resulting in $e_{\theta_{1}}<10^{\frac{-160}{20}}$.
} 


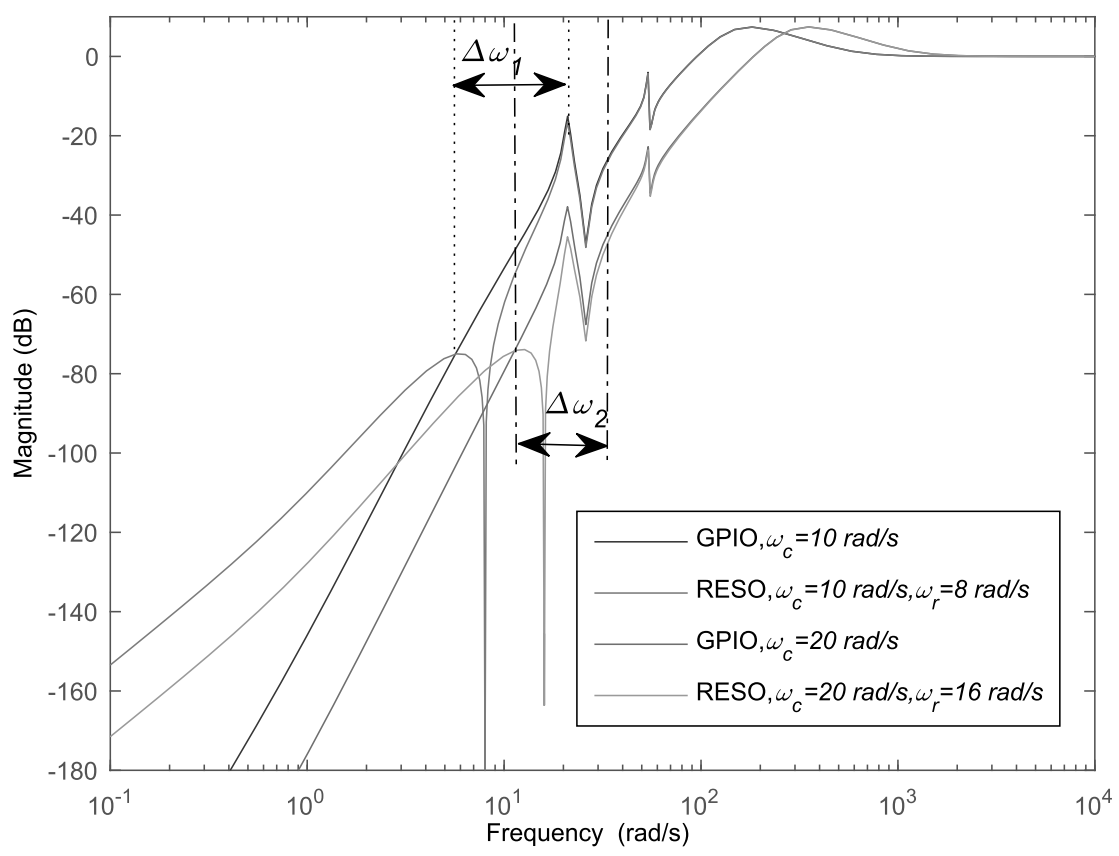

Fig. 5. Magnitude frequency responses of transfer function $G_{e}(s)$.

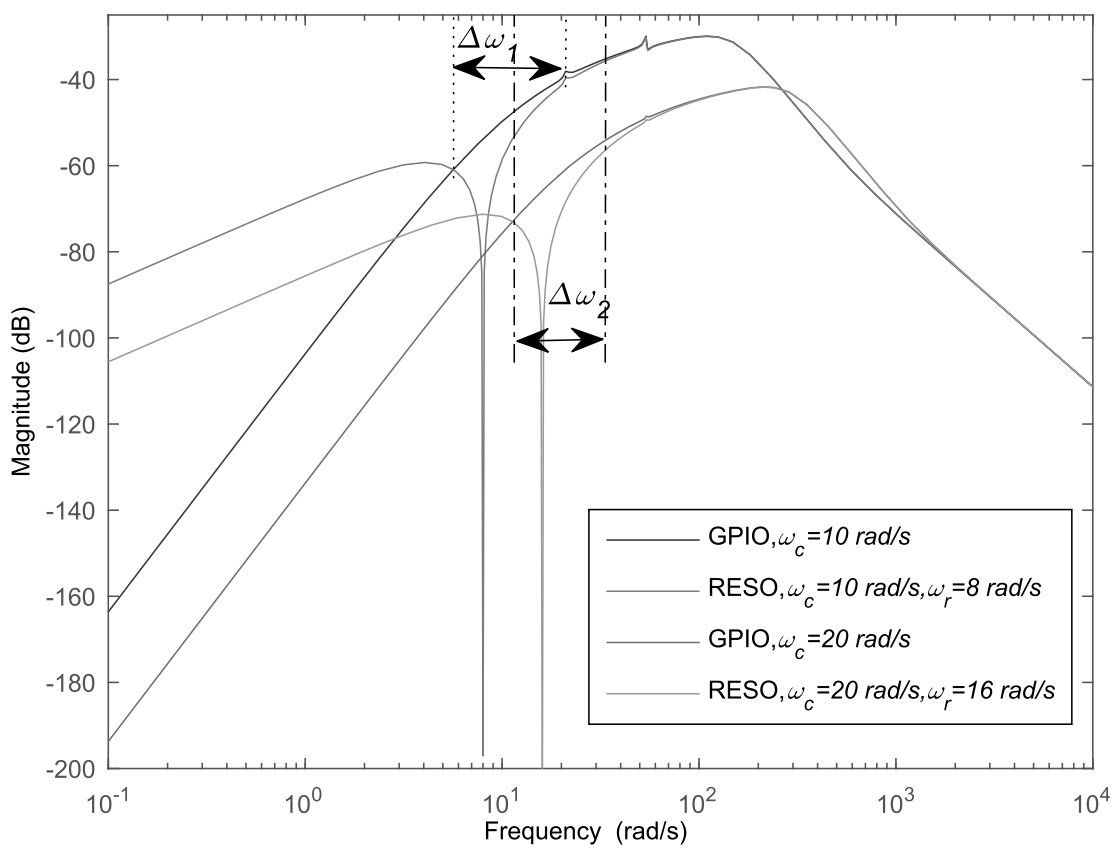

Fig. 6. Magnitude frequency responses of transfer function $G_{d}(s)$.

\section{Experimental validation}

In order to verify the efficacy of the proposed error-based control structure in dealing with harmonic uncertainty, several experimental tests have been conducted. The tests have been performed in a hardware-in-the-loop setup, in which the control algorithm was implemented in a Matlab/Simulink environment with sampling frequency of $T_{s}=1 \mathrm{kHz}$. The utilized control system was comprised of three subsystems: the mechanism (including motor and sensors), the real-time 


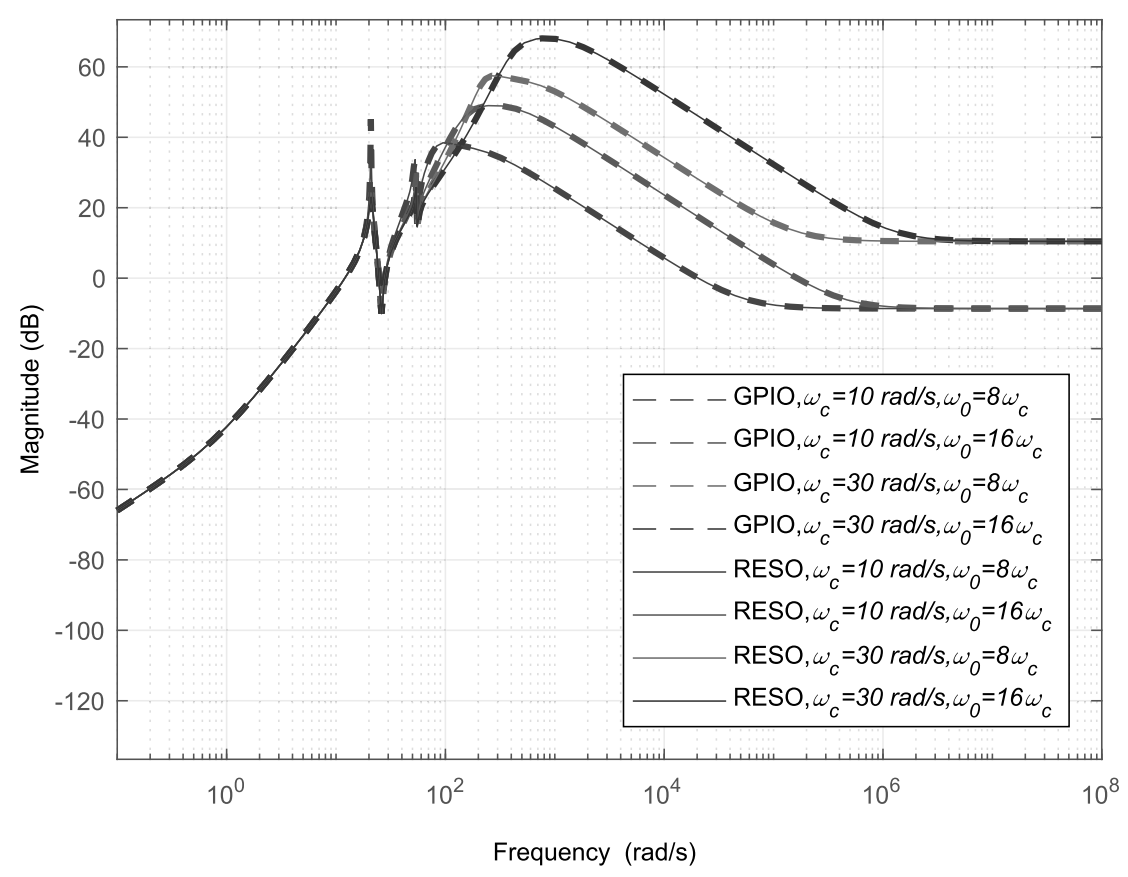

Fig. 7. Magnitude frequency responses of transfer function $G_{n}(s)$.

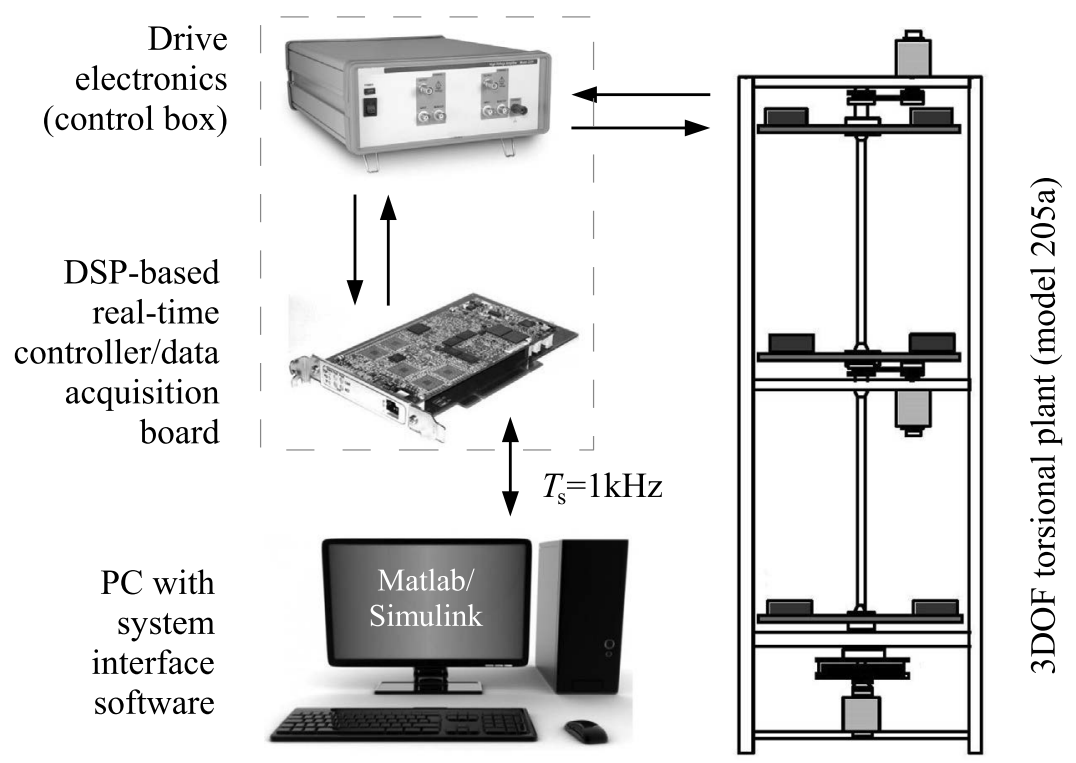

Fig. 8. A functional overview of the used real-time control system.

controller/drive electronics, and the user/system ("executive") interface software. Such architecture, illustrated in Fig. 8, is consistent with modern industrial control implementation. The experimental setup is a commercially available testbed M205a from ECP, ${ }^{6}$ details of which can be found in [28].

\subsection{Methodology}

The methodology behind the performed multi-criteria control evaluation assumed the execution of following tests: 
-Expl: establishing nominal performance,

-Exp2: robustness test against parametric uncertainty,

-Exp3: robustness test against external disturbance,

-Exp4: quantitative comparison with standard solution:

-Exp4a: results of standard GPIO-based control,

-Exp4b: results of proposed RESO-based control,

-Exp4c: direct comparison of $\operatorname{Exp} 4 a$ and $\operatorname{Exp} 4 b$.

In the experiment $\operatorname{Expl}$, the nominal performance of the proposed control structure is established. Since the characteristics of the lumped disturbance is assumed to be unknown (which is often the case in practice), the error-based RESO is implemented with an approximated frequency of harmonic uncertainty $\hat{\omega}_{\mathrm{r}} \approx \omega_{\mathrm{r}}$. Its rough value $\left(\hat{\omega}_{\mathrm{r}}=2.44 \mathrm{~Hz}\right)$ is obtained from a power spectral density (PSD) analysis, conducted a'priori during a single open-loop system run, in which the system controlled output $\left(\theta_{1}\right)$ has been used as an input for the analysis. Both the used input profile and the obtained outcome of the PSD analysis are depicted in Fig. 9. Following general ADRC tuning guidelines from [41], the desired observer and controller bandwidths are set as $\omega_{0}=150 \mathrm{rad} / \mathrm{s}$ and $\omega_{c}=8 \mathrm{rad} / \mathrm{s}$, respectively. In practice, selection of $\omega_{c}$ results from a compromise

a)

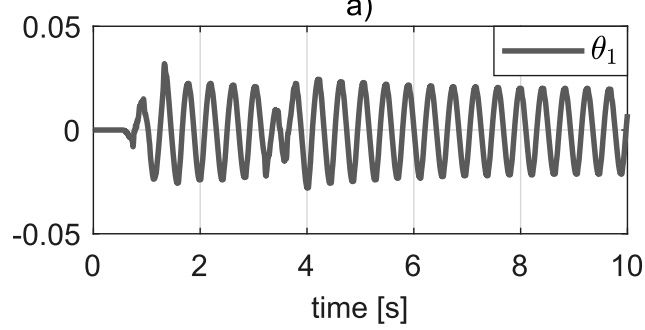

b)

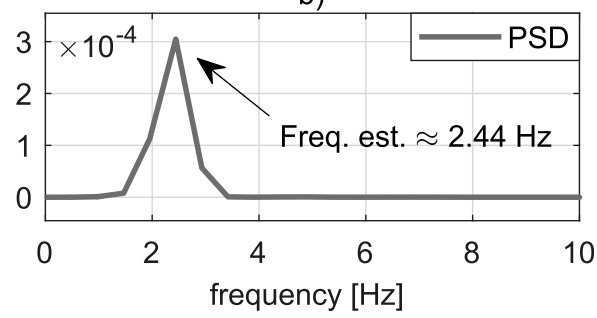

Fig. 9. [Expl] Estimation of input signal frequency (a) using the PSD analysis (b).

a)

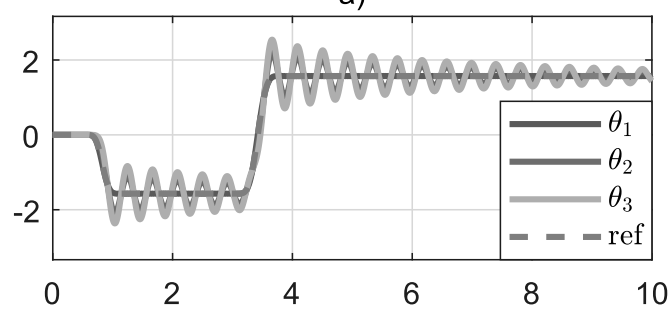

c)
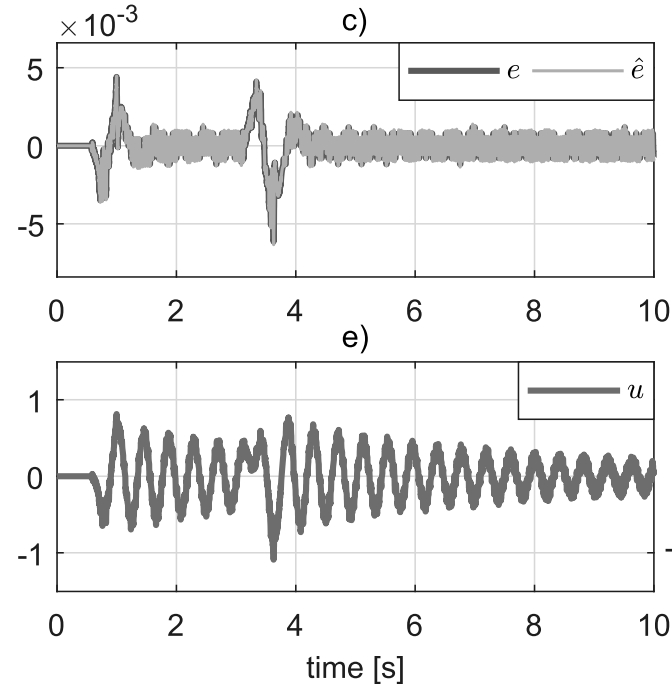

b)

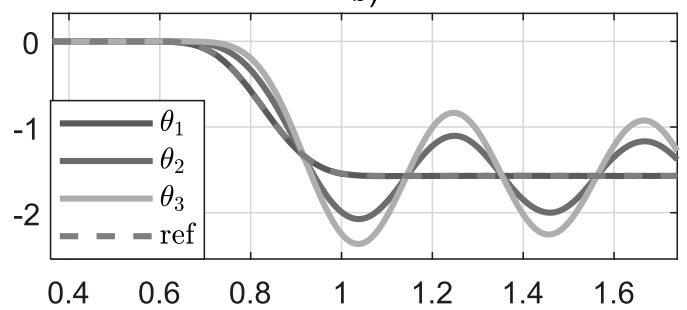

d)
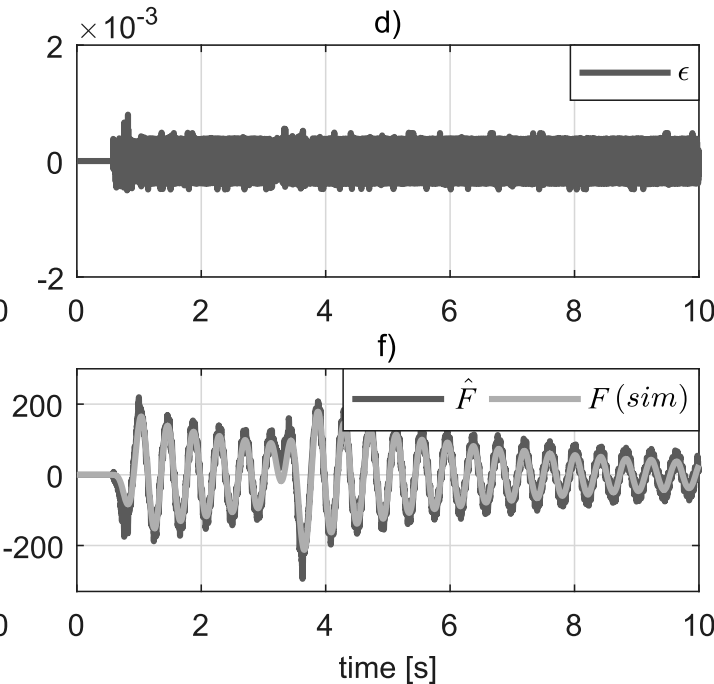

Fig. 10. [Expl] Results of the proposed error-based ADRC structure with RESO in terms of: angular discs positions *(a), with its zoomed-in version for better legibility (b), feedback error and its estimation from RESO (c), estimation error (d), control signal (e), and estimated total disturbance compared with the "real" total disturbance obtained in a simulated environment (f). 
between a desired decay-time for the output error (larger $\omega_{c}$ ) and such factors as a limited admissible amplitude of a control signal, the presence of a high-frequency noise in a feedback loop, and a limited sampling frequency when the control system is implemented in discrete-time. On the other hand, the observer bandwidth $\omega_{0}$ should be always wider relative to the control bandwidth determined by $\omega_{c}$. It is worth to emphasize that the proposed forms of error-based RESO gains have been computed upon the prescribed structure of polynomial $P_{o}^{*}(s) \triangleq\left(s+\omega_{0}\right)^{5}$ from $(22)$, which seems especially justified under conditions when a sharp frequency separation between the low-frequency content of the output error and a highfrequency content of the measurement noise is possible. In other circumstances, one can replace the form of polynomial $P_{o}^{*}(s)$ and the forms of error-based RESO gains by appropriately optimized formulas. The established nominal case in Expl is used as a point of reference for upcoming robustness tests (Exp2, Exp3) and comparison with GPIO-based technique (Exp4). From that point on, no additional observer/controller retuning is performed.

In the experiment $\operatorname{Exp} 2$, the performance of the proposed governor in dealing with uncertainty in user-defined selection of signal frequency $\omega_{\mathrm{r}}$ is evaluated. This test emulates a scenario in which just a rough value of $\omega_{\mathrm{r}}$ is known. The feedback errors are compared for three cases, namely for the nominal $\hat{\omega}_{r}$ (obtained in Expl) as well as its artificially decreased $\left(90 \% \hat{\omega}_{r}\right)$ and increased $\left(110 \% \hat{\omega}_{r}\right)$ values. In the same experiment, the performance of the introduced control structure in dealing with uncertainty in selecting $b_{0}$ is evaluated. This test emulates a scenario where only an approximated value of $b_{0}$ is known and thus can be used for controller synthesis. It verifies the robustness of the used disturbance observer, as the uncertainty in the system gain is also the part of the total disturbance term (cf. (7)). The quality of trajectory tracking, understood in terms of obtained feedback errors, is compared for three cases, namely for the nominal $b_{0}$ (used in $\operatorname{Exp} I$ ) as well as its artificially decreased $\left(75 \% b_{0}\right)$ and increased $\left(125 \% b_{0}\right)$ value.

In the experiment $\operatorname{Exp} 3$, the performance of the proposed control scheme is investigated in dealing with unmodeled external disturbance, here defined as an extra torque being applied at different levels (discs) of the torsional plant. This test emulates a scenario in which there is extra dynamics in the system, which has not been taken into consideration during system tuning in Expl. The quality of trajectory tracking, being function of corresponding feedback errors, is compared in four cases, first one being the nominal case (no external disturbance) and in three cases where external disturbance is applied to: top, middle, and bottom disc, respectively.

In the experiment $\operatorname{Exp} 4$, a comparison of the proposed method with GPIO-based scheme is conducted. In order to make the comparison fair, a set of GPIOs is used that utilizes different pole-placement-based tuning strategies, namely: moderate (nominal bandwidth: $\omega_{0}=150 \mathrm{rad} / \mathrm{s}$ ), conservative (decreased bandwidth: $66 \% \omega_{0}$ ), and aggressive (increased bandwidth:

a)

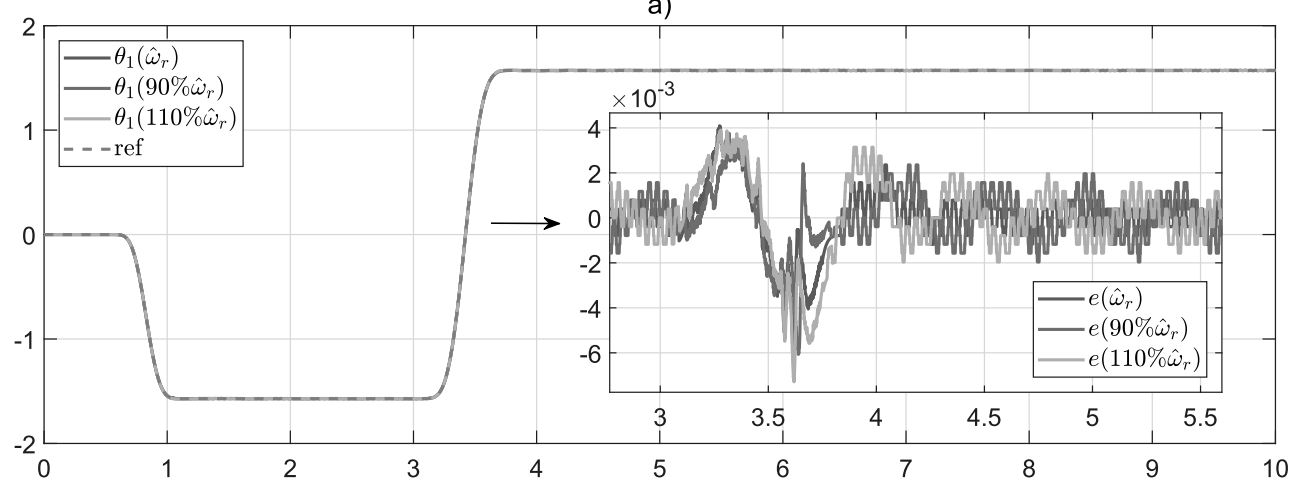

b)

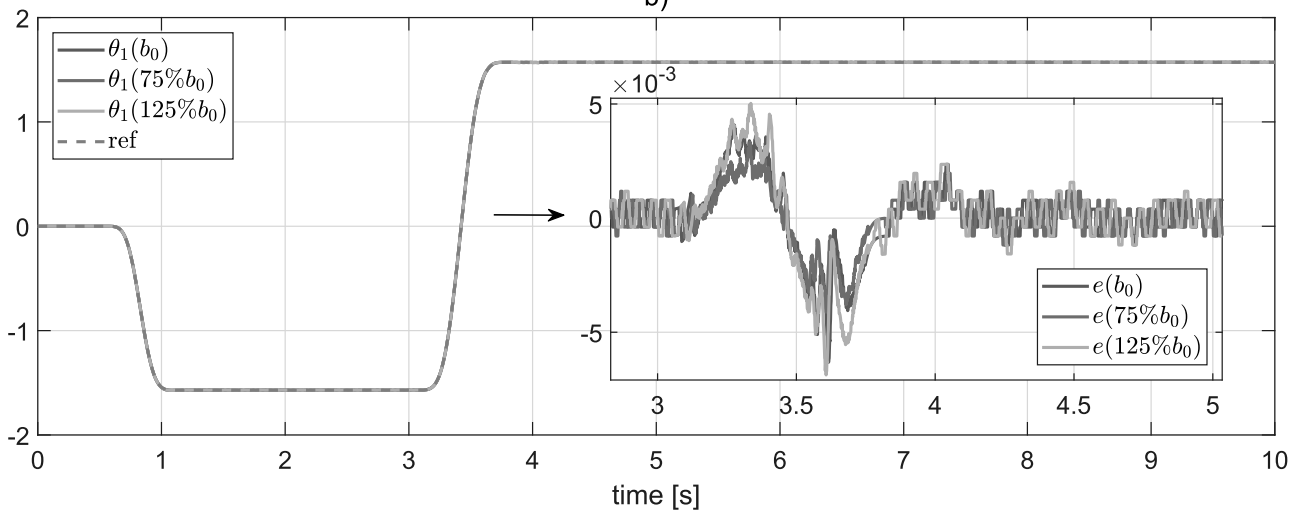

Fig. 11. [Exp2] Accuracy of trajectory tracking under different selection of frequency $\hat{\omega}_{r}(\mathrm{a})$ and system gain $b_{0}$ (b). 
a)
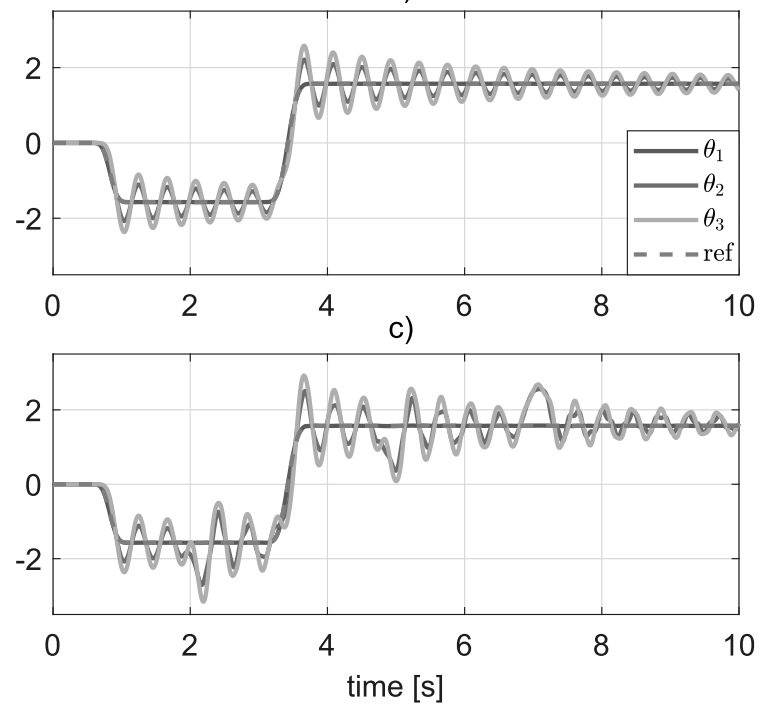

b)
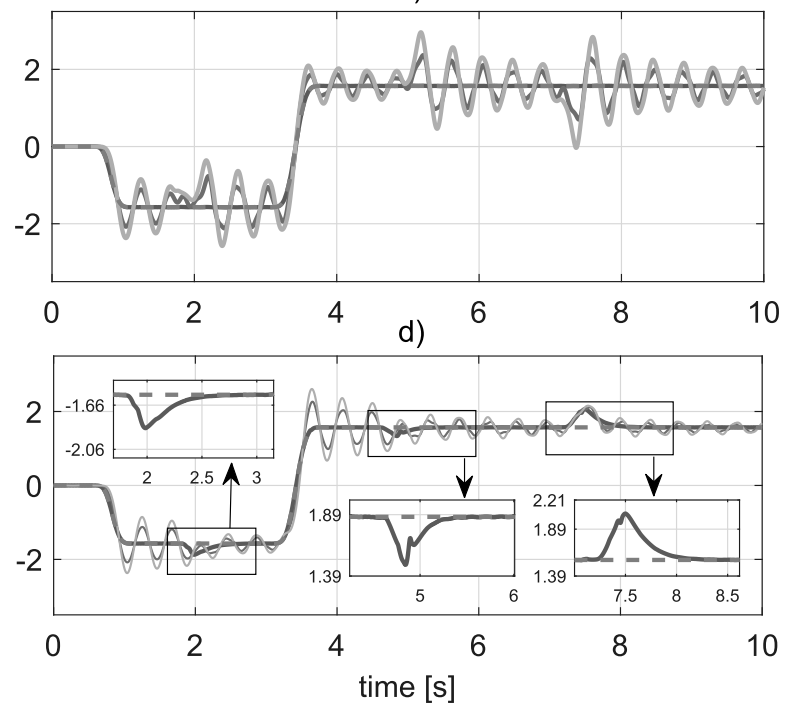

Fig. 12. [Exp3] Accuracy of trajectory tracking in case of no extra load disturbance (a) and in cases of the load placed at the top (b), middle (c), and bottom (d) disc.

$\left.133 \% \omega_{0}\right)$. The moderate tuning is deliberately set the same as the nominal case of the error-based RESO from Expl to make a common base for comparison.

\subsection{Experimental results}

The results of experiment Expl with the nominal case are gathered collectively in Fig. 10 . The realization of trajectory tracking is depicted in subplot (a) and its zoomed-in version in (b). The control objective is realized with satisfactory performance, which is also confirmed by resultant feedback error $\left(e \equiv e_{\theta_{1}}\right)$ in (c). Same subplot shows high quality of observer estimation which results in $\epsilon \triangleq e-\hat{e}$, seen in (d), being in close vicinity of zero. The generated control action $(u \equiv \tau)$ in (e) does not exceed physical limitations of the system and is practically acceptable, despite the presence of noise (being the byproduct of observer/controller tuning methodology), which does not noticeably influence system output. Additionally, since the actual total disturbance is not available through measurement in the considered experiment, its estimation is additionally compared in (f) with the total disturbance modeled in a dedicated simulation. This, alongside observer error convergence seen in (c) and (d), validates the correctness of the proposed error-based RESO design.

The outcomes of test $\operatorname{Exp} 2$ are depicted in Fig. 11. It can be seen that despite the artificially introduced uncertainty in $\hat{\omega}_{r}$ selection (compared to the value identified for the purpose of test Expl), the tracking performance, in terms of obtained feedback error signal (i.e. zoomed in plot), did not suffer noticeably. Although the proposed control scheme is robust, the level of its parametric robustness is limited, hence more significant mismatch in $\hat{\omega}_{r}$ selection would result at some point in worsening of the closed-loop performance, since instead of unburdening the observer as a partial modeling information about the controlled plant, the injected incorrect term $\hat{\omega}_{r}$ would introduce more uncertainty. Same test Exp2 also showed that the quality of target trajectory realization, in terms of resultant feedback error (i.e. zoomed in plot), remained at similar level when the artificially introduced parametric mismatch in $b_{0}$ is applied (in relation to the nominal value established in $\operatorname{Expl).}$

The outcomes of experiment $\operatorname{Exp} 3$ are gathered in Fig. 12. From the plots, it can be concluded that, when compared to the nominal case of no external disturbance in (a), the presence of additional, unmodeled dynamics in top and middle discs (cases (b) and (c), respectively) did influence visibly corresponding profiles $\theta_{2}$ and $\theta_{3}$ but did not influence the task realization in a significant way (as $\theta_{1} \rightarrow \theta_{1}^{*}$ ). The application of extra torque in the bottom disc, depicted in (d), had however visible unwanted effect on the system output. ${ }^{7}$ The influence of such unmodeled external interference, thanks to the utilized on-line disturbance observer-based control scheme, is effectively estimated and compensated in a timely manner (as highlighted by the zoomed in plots).

The results of experiment $\operatorname{Exp} 4$ are depicted in Figs. 13-15. First, in $\operatorname{Exp} 4 \mathrm{a}$, the GPIO-centered control scheme was evaluated using different tuning strategies. Fig. 13 shows quality trajectory tracking, resultant feedback error, GPIO estimation error, and control effort, respectively. From the plots, one can notice that the aggressive tuning results in the trajectory being

\footnotetext{
${ }^{7}$ This effect makes sense from the mechanical point of view, since in this case, the load has been placed near the actuated degree-of-freedom, hence its effect on the output is the most significant among the considered scenarios.
} 

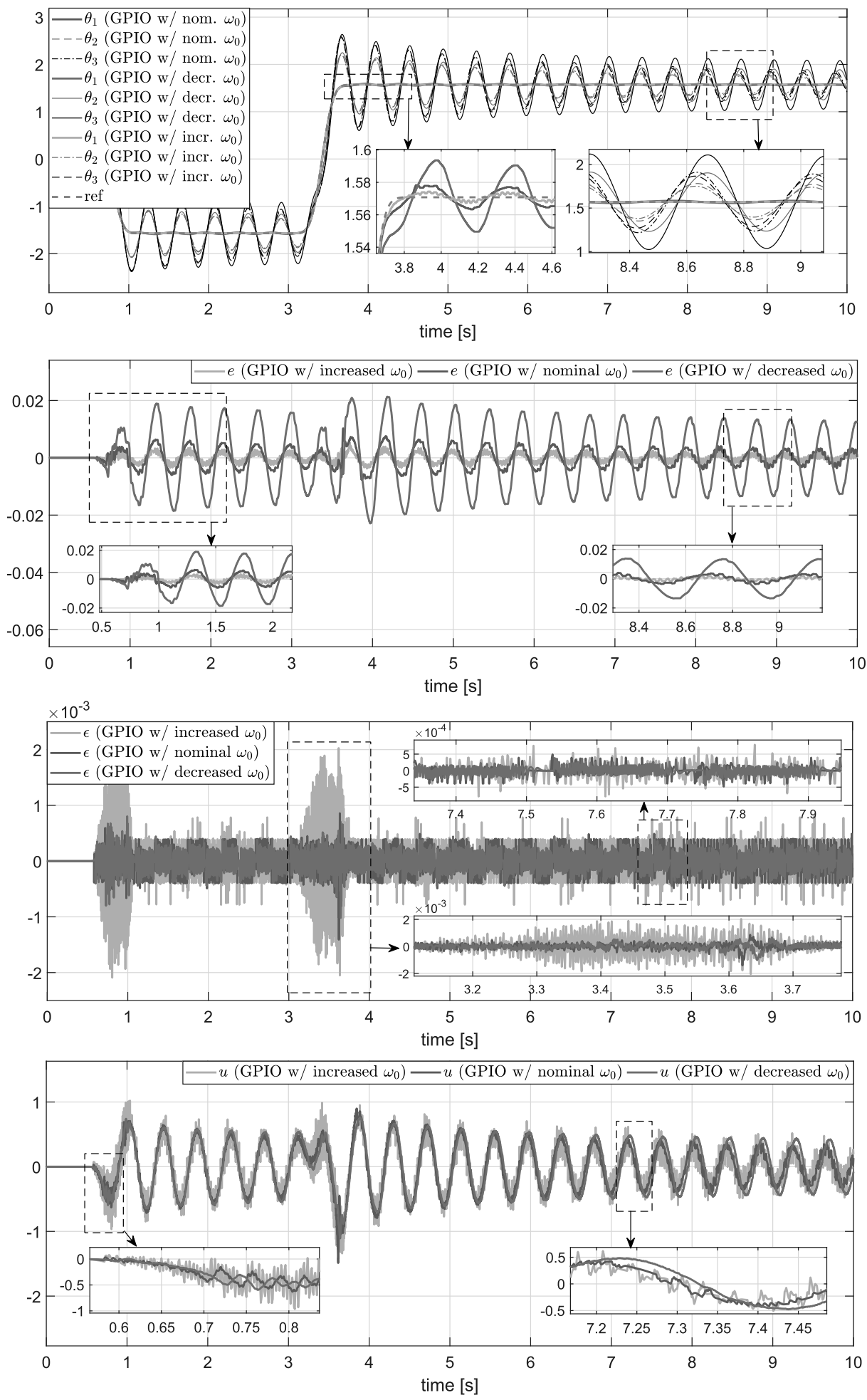

Fig. 13. [Exp4a] Results of conventional GPIO-based control scheme in terms of (from the top): angular discs positions $\left(\theta_{1}-\theta_{3}\right)$, feedback error $(e)$, estimation error $(\epsilon)$, and control signal $(u)$.

tracked more accurately but at the same time, due to noise amplification in the observer, the control signal contains more high-frequency noise components. Opposite conclusions can be made when analyzing the results obtained with conservative tuning, namely lower observer bandwidth solved the problem of noise over-amplification but resulted in worsening of the 

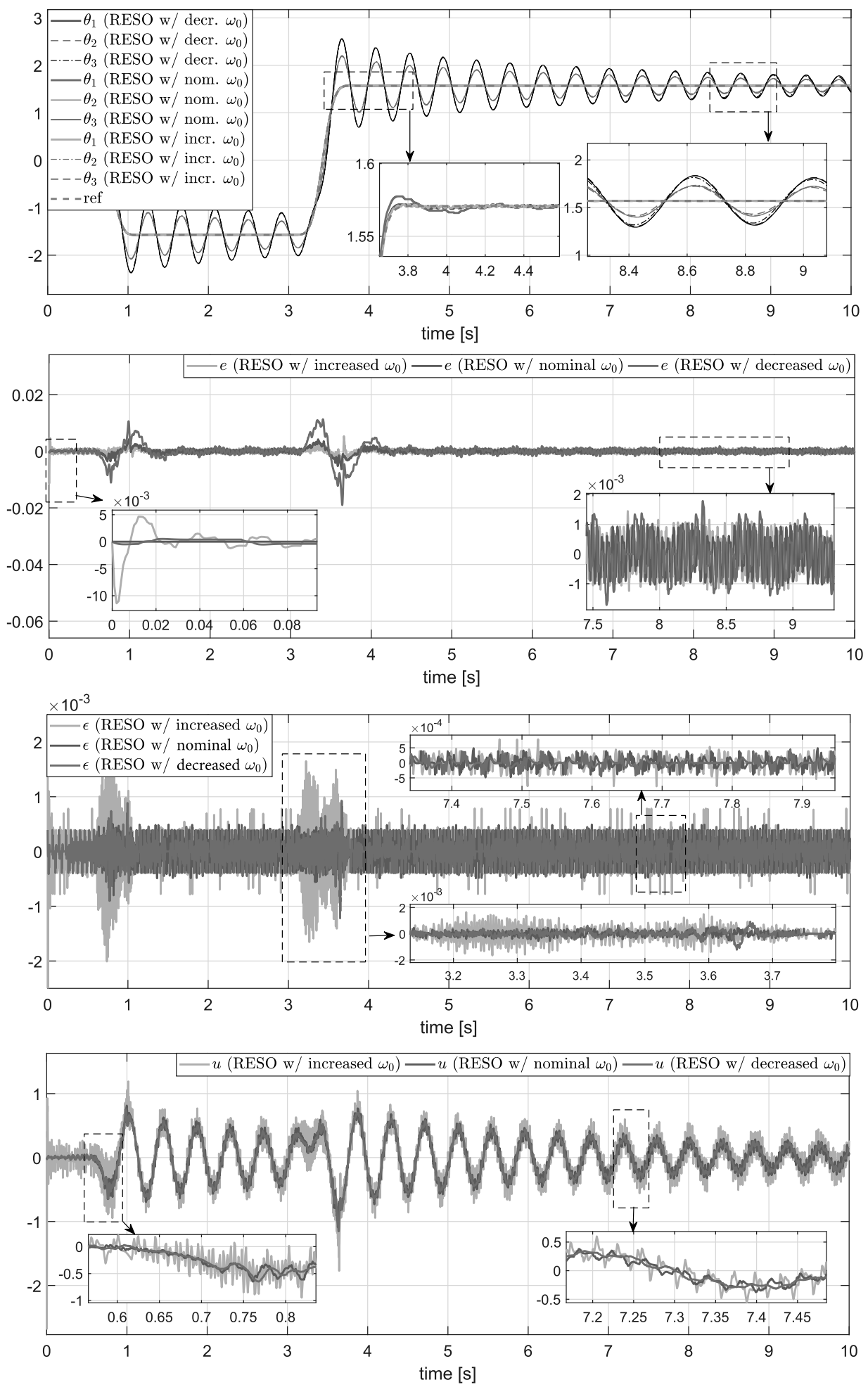

Fig. 14. [Exp4b] Result of proposed RESO-based control scheme in terms of (from the top): angular discs positions $\left(\theta_{1}-\theta_{3}\right)$, feedback error $(e)$, estimation error $(\epsilon)$, and control signal $(u)$.

tracking precision. This relation is well-known in observer-based control algorithms and has been studied to date also in the context of GPIO (for details see [34,35]). Here, it means that it is possible to compensate polynomial-type uncertainty $\left(F_{p}(t)\right.$ ) effectively using GPIO, but to reject fast harmonic-type perturbation $\left(F_{w}(t)\right)$ it is necessary to increase the number $m$ of 
a)

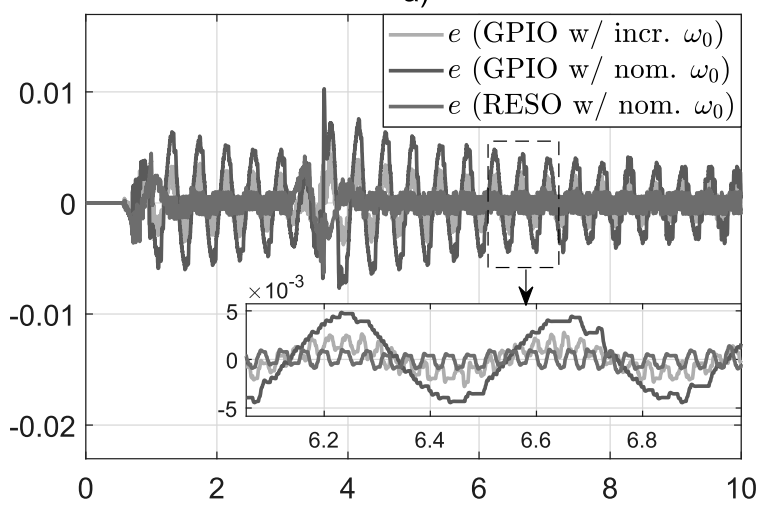

c)

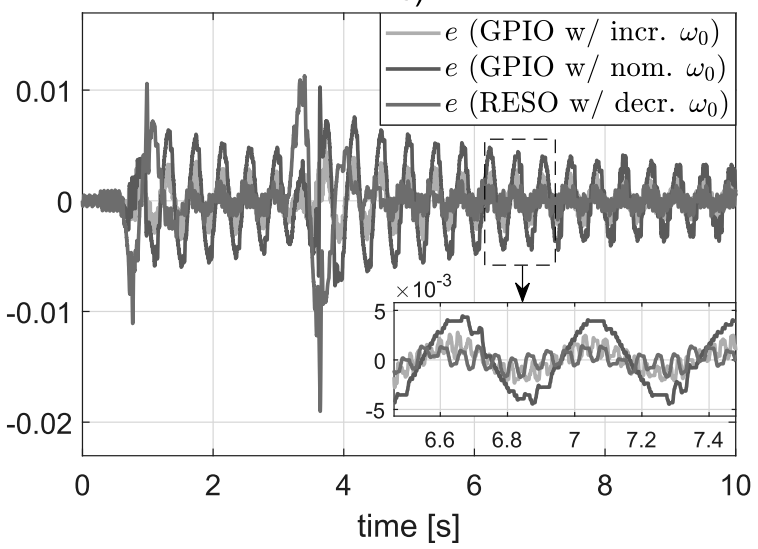

b)

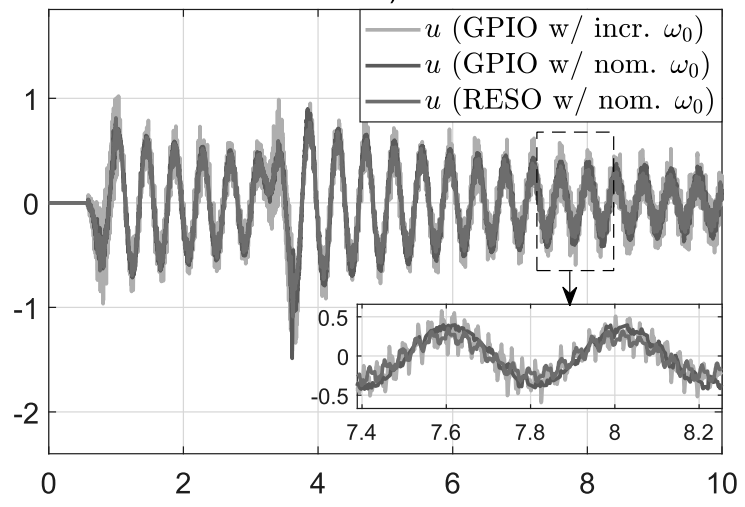

d)

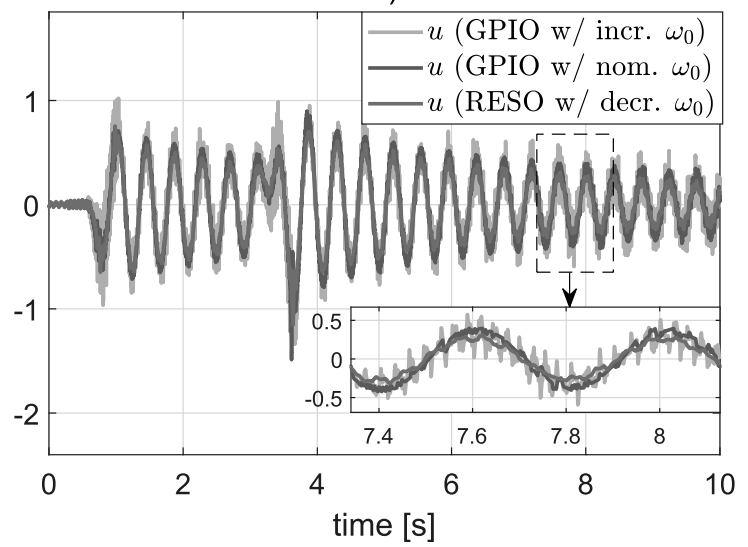

Fig. 15. [Exp4c] Direct comparison of conventional GPIO- and proposed RESO-based control schemes in terms of feedback error (e) and control signal ( $u$ ).

extended states in GPIO or use high observer bandwidth $\left(\omega_{0}\right)$. By doing so, the GPIO is more sensitive to measurement noise (due to larger observer gains), which leads to degradation of the control signal (11). It usually comes down to a compromise that has to be made between tracking accuracy and control action profile. It is thus desirable to have a control technique that would go beyond this compromise and provide high-precision tracking with reasonable energy consumption profile. For that reason, the proposed error-based ADRC with RESO was tested next $(\operatorname{Exp} 4 \mathrm{~b})$ and its results are gathered in Fig. 14. Similar tests to $\operatorname{Exp} 4$ a have been conducted here, which utilized different tuning approaches. From the figures, one can notice that the aggressive tuning here also results in the trajectory being tracked more accurately with the noise being amplified in the observer and the control signal containing noises as a result. ${ }^{8}$ However, when compared to the results of GPIO-based scheme (for corresponding tuning strategies), the control accuracy is quantitatively higher for RESO-based solution, while the noise amplification in the observer, and consequently the control signal, are smaller.

This calls for a cross-comparison of GPIO- and RESO-based techniques with different tuning strategies, which has been performed here and its outcomes are depicted in Fig. 15. From the top figures, it can be noticed that the GPIO-based scheme (with aggressive tuning) still did perform worse than the RESO-based scheme (with just moderate tuning). It is seen in terms of both feedback errors (a) and energy efficiency (b). Furthermore, even the results obtained with the use of RESO-based approach with conservative tuning show better tracking in steady state than GPIO-based method with either moderate or aggressive tuning (c), while keeping the control signal at similar level in those cases (d).

By analyzing the results, it can be deduced that the combination of polynomial and sinusoidal disturbance models in the proposed error-based ADRC with RESO increases the quality of partial modeling information about the working environment, thus unburdens the user as it decreases the overall system uncertainty the observer has to reconstruct in each time instant. It has direct consequence on the convergence of the estimation error, which leads to the speed and accuracy of online total disturbance reconstruction and rejection being the back-bone of the proposed observer-centered technique. In the introduced control framework, the sinusoidal disturbance model is elegantly incorporated in the error-based RESO, which overcomes one of the most common problems of observer-based controllers for highly uncertain systems, being the measurement noises amplification due to high gains.

\footnotetext{
${ }^{8}$ Such outcome was expected due to the fundamental structure of the observer in which the observer error, containing the system output inevitably subjected to sensor noise, is amplified by the user-selected tuning gains.
} 


\subsection{Discussion on potential applications and further development}

The future work related to the introduced control method will revolve around two aspects. One is to focus on further increasing the tracking performance through introduction of an on-line resonant frequency estimation (e.g. [42,43]) as well as observers with finite-time convergence (e.g. [44]). The second one is expanding the applicability range of proposed control approach scheme to different systems. Several target scenarios are considered, including high-order systems (e.g. buck converter-fed DC motor) and systems with states and inputs constraints [45].

\section{Conclusions}

In this article, a new practically appealing control structure for tackling harmonic uncertainties has been proposed and exemplified using a laboratory benchmark system. Vibration suppression has been formulated as a fundamental problem of disturbance rejection, for which a tailored-made ADRC scheme has been conceived. The introduced governing framework utilizing a resonant version of an extended state observer was shown to be an effective tool for real-time estimation and then compensation of sinusoidal-type uncertainties. Its expression in industry familiar error-based form made it easily implementable across different control platforms. The utilized methodology introduced a significant simplification to control synthesis and tuning without the loss of robustness, adaptability, and intuitiveness of the original output-based ADRC design. It also eliminated the common assumption about availability of higher order target derivatives, thus making the use of extra sensors or differentiators obsolete.

\section{Declaration of Competing Interest}

The authors declare that they have no known competing financial interests or personal relationships that could have appeared to influence the work reported in this paper.

\section{Acknowledgment}

This work was supported in part by the National Natural Science Foundation of China under the Grants 61973080, 61750110525 , and 61633003.

\section{Appendix A. Plant transfer functions coefficients}

$$
\begin{aligned}
& a_{41}=J_{1} J_{2} J_{3}, a_{42}=J_{1} J_{2} c_{3}+J_{1} J_{3} c_{2}+J_{2} J_{3} c_{1}, \\
& a_{43}=J_{1}\left(J_{2} \kappa_{2}+J_{3} \kappa_{1}+J_{3} \kappa_{2}+c_{2} c_{3}\right),+J_{2}\left(J_{3} \kappa_{1}+c_{1} c_{3}\right)+J_{3} c_{1} c_{2}, \\
& a_{44}=J_{1}\left(c_{2} \kappa_{2}+c_{3} \kappa_{1}+c_{3} \kappa_{2}\right)+J_{2}\left(c_{1} \kappa_{2}+c_{3} \kappa_{1}\right),+J_{3}\left(c_{1} \kappa_{1}+c_{1} \kappa_{2}+c_{2} \kappa_{1}\right)+c_{1} c_{2} c_{3}, \\
& a_{45}=\left(J_{1}+J_{2}+J_{3}\right) \kappa_{1} \kappa_{2},+c_{1}\left(c_{2} \kappa_{2}+c_{3} \kappa_{1}+c_{3} \kappa_{2}\right)+c_{2} c_{3} \kappa_{1}, \\
& a_{46}=\left(c_{1}+c_{2}+c_{3}\right) \kappa_{1} \kappa_{2}, \\
& a_{11}=J_{2} J_{3}, a_{12}=J_{2} c_{3}+J_{3} c_{2}, \\
& a_{13}=J_{2} \kappa_{2}+c_{2} c_{3}+J_{3} \kappa_{1}+J_{3} \kappa_{2}, \\
& a_{14}=c_{2} \kappa_{2}+c_{3} \kappa_{1}+c_{3} \kappa_{2}, a_{15}=\kappa_{1} \kappa_{2}, \\
& a_{21}=\kappa_{1} J_{3}, a_{22}=\kappa_{1} c_{3}, a_{23}=\kappa_{1} \kappa_{2}, a_{31}=\kappa_{1} \kappa_{2} .
\end{aligned}
$$

with nominal values of the torsional plant being $J_{1}=0.0037, J_{2}=0.0011, J_{3}=J_{2}, \kappa_{1}=1.3, \kappa_{2}=1.2$, as well as $c_{1}=0.002, c_{2}=0.0015, c_{3}=c_{2}$ which are the generalized friction coefficients related to the motions of first, second, and third disc, respectively.

\section{References}

[1] S. Zhao, Z. Gao, An active disturbance rejection based approach to vibration suppression in two-inertia systems, Asian J. Control 15 (2013) 350-362.

[2] H. Zhu, Y. Li, W. Shen, S. Zhu, Mechanical and energy-harvesting model for electromagnetic inertial mass dampers, Mech. Syst. Signal Process. 120 (2019) 203-220.

[3] I. Golovin, S. Palis, Robust control for active damping of elastic gantry crane vibrations, Mech. Syst. Signal Process. 121 (2019) $264-278$.

[4] X. Zhao, J. Huang, Distributed-mass payload dynamics and control of dual cranes undergoing planar motions, Mech. Syst. Signal Process. 126 (2019) 636-648.

[5] G. Wang, J. Tan, Z. Zhao, S. Cui, H. Wu, Mechanical and energetic characteristics of an energy harvesting type piezoelectric ultrasonic actuator, Mech. Syst. Signal Process. 128 (2019) 110-125.

[6] J. Zou, X. Guo, M.A.A. Abdelkareem, L. Xu, J. Zhang, Modelling and ride analysis of a hydraulic interconnected suspension based on the hydraulic energy regenerative shock absorbers, Mech. Syst. Signal Process. 127 (2019) 345-369.

[7] W. Sun, H. Gao, B. Yao, Adaptive robust vibration control of full-car active suspensions with electrohydraulic actuators, IEEE Trans. Control Syst. Technol. 21 (6) (2013) 2417-2422.

[8] C. Chen, Z. Liu, Y. Zhang, C.L.P. Chen, Modeling and adaptive compensation of unknown multiple frequency vibrations for the stabilization and control of an active isolation system, IEEE Trans. Control Syst. Technol. 24 (3) (2016) 900-911.

[9] W. He, S.S. Ge, Vibration control of a flexible beam with output constraint, IEEE Trans. Ind. Electron. 62 (8) (2015) $5023-5030$. 
[10] Y. Pu, H. Zhou, Z. Meng, Multi-channel adaptive active vibration control of piezoelectric smart plate with online secondary path modelling using PZT patches, Mech. Syst. Signal Process. 120 (2019) 166-179.

[11] J. Wang, Active vibration control: a graphical approach for optimal distribution, Mech. Syst. Signal Process. 124 (2019) $632-642$.

[12] J. Han, From PID to active disturbance rejection control, IEEE Trans. Ind. Electron. 56 (3) (2009) $900-906$.

[13] G. Wu, L. Sun, K.Y. Lee, Disturbance rejection control of a fuel cell power plant in a grid-connected system, Control Eng. Practice 60 (2017) 183-192.

[14] J. Yang, H. Cui, S. Li, A. Zolotas, Optimized active disturbance rejection control for DC-DC buck converters with uncertainties using a reduced-order GP observer, IEEE Trans. Circuits Syst. 99 (2017) 1-10.

[15] H. Sira-Ramirez, A. Luviano-Juárez, M. Ramírez-Neria, E.W. Zurita-Bustamante, Active Disturbance Rejection Control of Dynamic Systems: A Flatness Based Approach, Butterworth-Heinemann, 2018.

[16] J. Yang, W.H. Chen, S. Li, L. Guo, Y. Yan, Disturbance/uncertainty estimation and attenuation techniques in PMSM drives - A survey, IEEE Trans. Ind. Electron. 64 (4) (2017) 3273-3285.

[17] W. Xue, R. Madonski, K. Lakomy, Z. Gao, Y. Huang, Add-on module of active disturbance rejection for set-point tracking of motion control systems, IEEE Trans. Ind. Appl. 53 (4) (2017) 4028-4040.

[18] Q. Zheng, L. Dong, D.H. Lee, Z. Gao, Active disturbance rejection control for MEMS gyroscopes, IEEE Trans. Control Syst. Technol. 17 (6) (2009) 14321438.

[19] K. Song, T. Hao, H. Xie, Disturbance rejection control of air-fuel ratio with transport-delay in engines, Control Eng. Practice 79 (2018) 36-49.

[20] R. Madonski, M. Nowicki, P. Herman, Practical solution to positivity problem in water management systems - an ADRC approach, American Control Conference, 2016, pp. 1542-1547.

[21] J.S. Bang, H. Shim, S.K. Park, J.H. Seo, Robust tracking and vibration suppression for a two-inertia system by combining backstepping approach with disturbance observer, IEEE Trans. Ind. Electron. 57 (9) (2010) 3197-3206.

[22] Z. Wang, Y. Yan, J. Yang, S. Li, Q. Li, Robust voltage regulation of a DC-AC inverter with load variations via a HDOBC approach, IEEE Trans. on Circuits and Systems II: Express Briefs. 66 (7) (2019) 1172-1176.

[23] S. Li, J. Yang, W.H. Chen, X. Chen, Generalized extended state observer based control for systems with mismatched uncertainties, IEEE Trans. Ind. Electron. 59 (12) (2012) 4792-4802.

[24] W.H. Chen, Harmonic disturbance observer for nonlinear systems, J. Dyn. Syst. Meas. Contr. 125 (1) (2003) $114-117$.

[25] Y. Yan, J. Yang, Z. Sun, C. Zhang, S. Li, H. Yu, Robust speed regulation for PMSM servo system with multiple sources of disturbances via an augmented disturbance observer, IEEE/ASME Trans. Mechatronics 23 (2) (2018) 769-780.

[26] J. Yang, Z. Ding, W.H. Chen, S. Li, Output-based disturbance rejection control for non-linear uncertain systems with unknown frequency disturbances using an observer backstepping approach, IET Control Theory Appl. 10 (9) (2016) 1052-1060.

[27] M.R. Stankovic, M.R. Rapaic, S.M. Manojlovic, S.T. Mitrovic, S.M. Simic, M.B. Naumovic, Optimised active disturbance rejection motion control with resonant extended state observer, Int. J. Control (2017) 1-12.

[28] Torsional Control System, Manual for Model M205a, Educational Control Products (ECP), 1 Buckskin Court Bell Canyon, CA, USA

[29] L.M. Mierovitch, Elements of Vibration Analysis, McGraw-Hill Book Co., 1975.

[30] Z. Gao, On the centrality of disturbance rejection in automatic control, ISA Trans. 53 (4) (2014) $850-857$.

[31] M.M. Michalek, Robust trajectory following without availability of the reference time-derivatives in the control scheme with active disturbance rejection, American Control Conference, 2016, pp. 1536-1541.

[32] H. Zhang, Information Driven Control Design: A Case for PMSM Control Doctoral dissertation, Cleveland State University, 2017.

[33] Z. Gao, Scaling and bandwidth-parameterization based controller tuning, American Control Conference, vol. 6, 2003, pp. 4989-4996.

[34] A.A. Godbole, J.P. Kolhe, S.E. Talole, Performance analysis of generalized extended state observer in tackling sinusoidal disturbances, IEEE Trans. Control Syst. Technol. 21 (6) (2013) 2212-2223.

[35] R. Madonski, P. Herman, On the usefulness of higher-order disturbance observers in real control scenarios based on perturbation estimation and mitigation, in: International Workshop on Robot Motion and Control, 2013, pp. 252-257.

[36] S. Shao, Z. Gao, On the conditions of exponential stability in active disturbance rejection control based on singular perturbation analysis, Int. J. Control 90 (10) (2017) 2085-2097.

[37] P. Kokotovic, H.K. Khalil, J. O’Reilly, Singular Perturbation Methods in Control: Analysis and Design, SIAM, 1999.

[38] R. Madonski, S. Shao, H. Zhang, Z. Gao, J. Yang, S. Li, General error-based active disturbance rejection control for swift industrial implementations, Control Eng. Practice 84 (2019) 218-229.

[39] L.C. Evans, R.F. Gariepy, Measure Theory and Fine Properties of Functions, CRC Press, 1991.

[40] K.J. Astrom, R.M. Murray, Feedback Systems: An Introduction for Scientists and Engineers, Princeton University Press, 2010.

[41] R. Madonski, P. Herman, Survey on methods of increasing the efficiency of extended state disturbance observers, ISA Trans. 56 (2015) 18-27.

[42] Y. Yan, J. Yang, S. Li, J. Sun, Adaptive composite disturbance observer based control for PMSM subject to multiple disturbances, Proc. Chinese Control Conference, 2016, pp. 4751-4756.

[43] R. Madonski, Z. Gao, K. Lakomy, Towards a turnkey solution of industrial control under the active disturbance rejection paradigm, Proc. Conference of the Society of Instrument and Control Engineers of Japan, 2015, pp. 616-621.

[44] H. Pan, W. Sun, H. Gao, X. Jing, Disturbance observer-based adaptive tracking control with actuator saturation and its application, IEEE Trans. Autom. Sci. Eng. 13 (2) (2016) 868-875.

[45] W. Sun, Y. Liu, H. Gao, Constrained sampled-data ARC for a class of cascaded nonlinear systems with applications to motor-servo systems, IEEE Trans. Ind. Informatics 15 (2) (2019) 766-776. 